\title{
48. X-RAY MINERALOGY AND MINERAL GEOCHEMISTRY OF CENOZOIC STRATA (LEG 80) AND PETROGRAPHIC STUDY OF ASSOCIATED PEBBLES ${ }^{1}$
}

\author{
G. Chennaux and J. Esquevin, Société Nationale Elf-Aquitaine, Pau \\ A. Jourdan, Compagnie Française des Pétroles, Pessac \\ and \\ C. Latouche and N. Maillet, Institut de Géologie du Bassin d'Aquitaine, Université de Bordeaux I²
}

\begin{abstract}
Cenozoic sediments recovered from Sites 548, 549, and 550 were the objects of mineralogical (bulk sample and $<2-\mu \mathrm{m}$ fraction) and geochemical ( $\mathrm{HCl}$ extract) studies. Thin sections of rock pebbles embedded in sediments (upper levels at Site 548, particularly) were examined on a polarizing microscope.

This study outlines the vertical and lateral variation and evolution of the sedimentation. In the Paleocene and lower Eocene, the clay fraction is abundant and smectite is practically the sole existing clay mineral. $\mathrm{High} \mathrm{Mn}, \mathrm{Al}, \mathrm{Fe}, \mathrm{Mg}$, and $\mathrm{K}$ contents were measured in $\mathrm{HCl}$ extracts. Through the middle Eocene, carbonates become more abundant-highly dominant at Site 548. Metal contents in $\mathrm{HCl}$ extracts are very low. The clay fraction, although dominated at all sites by smectites, becomes richer in illite and poorly crystallized chlorite. At the middle/upper Miocene boundary, a significant decrease in the smectite/(illite + chlorite) ratio occurs at all sites, and this decrease continues into the middle Pliocene. This decrease is marked by an abrupt increase of quartz at Site 548. At the two other sites, carbonates remain highly predominant; $\mathrm{HCl}$ extracts reflect the relative abundance of the clay and carbonate fractions. After a brief recurrence of smectite in a high-metal-content interval, illite and chlorite become the dominant clay minerals in the upper Pliocene and the Pleistocene, where numerous variations in mineralogical composition occur in the clay fraction (Sites 548 and 549) or in non-clay components (Site 548). Several pebbles of various nature and origin, encountered in different levels of this interval at Site 548, appear to have an ice-rafting origin.

This study points out three main breaks in the general evolution of the sedimentation: the first, corresponding to the lower/middle Eocene boundary, is marked by the increase of carbonates and associated elements; the second, corresponding to the middle/upper Miocene boundary, is marked by a major decrease of the smectite/(illite + chlorite) ratio at all sites and by a massive appearance of quartz at Site 548; and the third, which occurred toward the late Pliocene, is marked by the dominance of primary clay minerals and the arrival of ice-rafted pebbles.

Our interpretation of results considers paleohydrological and paleoclimatic phenomena. It is suggested that the major middle/late Miocene break was associated with an increase of the deep bottom-water circulation between the Norwegian Sea and the North Atlantic Ocean, and/or a climatic evolution: humidification and cooling of climate. The changes toward the late Pliocene appear to have been the first effects of the glaciations at the end of Cenozoic.
\end{abstract}

\section{INTRODUCTION}

Previous mineralogical studies of sediments recovered at DSDP sites have provided numerous data on the evolution of the northeastern Atlantic deposits since the Mesozoic (Fan and Zemmels, 1972; Chamley et al., 1979; Latouche and Maillet, 1979; Cassat, 1979). Major modifications in mineralogical assemblages have been recognized in the Cenozoic, particularly between sediments deposited before and after the important hiatuses that often interrupt the stratigraphic series in the vicinity of the Paleogene/Neogene boundary (van Andel, 1975). These mineralogical changes have been interpreted by Latouche and Maillet $(1978,1980,1982)$ as regional consequences of the general paleoclimatic and paleohydrological evolution of the North Atlantic Ocean (Berg-

\footnotetext{
${ }^{1}$ Graciansky, P. C. de, Poag, C. W., et al., Init. Repts. DSDP, 80: Washington (U.S. Govt, Printing Office).

2 Addresses: (Chennaux and Esquevin) Société Nationale Elf-Aquitaine (Production) 64000 Pau, France; (Jourdan) Compagnie Française des Pétroles, 218-228 Avenue du HautLevêque, 33605 Pessac Cédex, France; (Latouche and Maillet) Institut de Géologie du Bassin d'Aquitaine, Université de Bordeaux I, 351 Cours de la Libération, 33405 Talence Cedex, France.
}

gren and Holister, 1974). The exact stratigraphic intervals in which these changes occurred have nevertheless not been well established. Leg 80 provides an opportunity to fill out previous data and interpretations as a consequence of fairly good recovery and of the critical location of the leg's drill sites with respect to the tropical-to-arctic climatic gradient.

Cenozoic sediments recovered at Sites 548, 549, and 550 were the objects of mineralogical and geochemical studies. X-ray diffraction was used in the mineralogical analysis of 103 bulk-sediment samples and later of 167 samples of fine-grained fractions $(<2 \mu \mathrm{m})$. Titration of $\mathrm{HCl}$ extracts was used in the geochemical analysis of three drilling-site samples. Rock pebbles occurring in the upper sediment levels of the three sites were examined on a polarizing microscope (13 samples). Both mineralogical and geochemical studies were carried out in the three collaborating laboratories with which the authors are affiliated: (1) Compagnie Française des Pétroles: Paleocene, Eocene, and Oligocene; clay minerals and pebble petrographic determination; (2) Institut de Géologie du Bassin d'Aquitaine: Miocene, Pliocene, and Pleistocene; clay minerals; (3) Société Nationale Elf-Aquitaine: total-sediment and geochemistry analyses. 


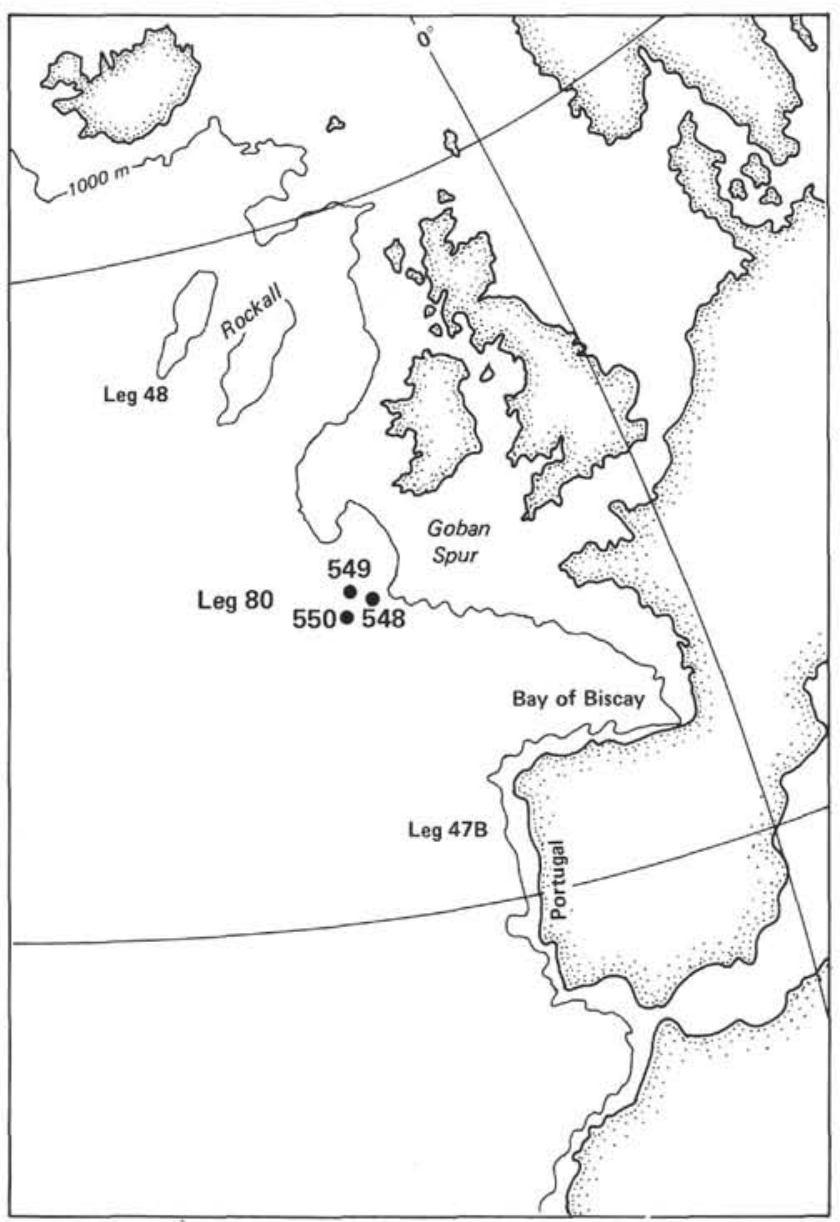

Figure 1. Location of Leg 80 relative to Legs 48 and 47B.

\section{MINERALOGICAL STUDY}

\section{METHODS}

\section{Bulk Sediments}

Percentages of quartz, calcite, and dolomite within the bulk sediment were determined by X-ray diffraction. Samples were crushed in an isogranulometric grinder. The diffractometer used was a Philips 1130 , and operating conditions were as follows: $\mathrm{Cu} \mathrm{k} \alpha$ radiation at 40 $\mathrm{kV}, 30 \mathrm{~mA}$; monochromator; Compton diffusion measurement for matrix-effect correction by computing program.

\section{Clay Fraction}

Bulk-sediment samples were dispersed in pure water using mechanical agitation. Samples with a high content of carbonates were first treated with $0.1 \mathrm{~N} \mathrm{HCl}$. After two or three washings in pure water, $<2-\mu \mathrm{m}$ subfractions were separated by gravity settling. The $<2-\mu \mathrm{m}$ portion was centrifuged, and the thick paste obtained was spread across two slides with a standard laboratory spatula. The first slide was saturated with ethylene glycol before analysis. The second was scanned untreated and then treated at $550^{\circ} \mathrm{C}$ for $1 \mathrm{hr}$. Minerals were identified on the basis of their typical reactions to classical treatment (Brown, 1961; Thorez, 1975). The different minerals were estimated semiquantitatively from diffractograms of the glycolated slides; their relative abundances in the clay fraction were evaluated on the basis of the height of their basal characteristic reflections. Experimental error was estimated to $\pm 10 \%$ (relative value), in accordance with the analysis of artificial mixtures of pure clay minerals. In the Eocene and Oligocene formations, kaolinites and chlorites have not been differentiated, 002 reflection of kaolinite and 004 of chlorite giving a nondefined peak in the vicinity of $3.56 \AA$.

\section{Analytical Data}

\section{Site 548 (Fig. 2, Table 1)}

Holes $548 \mathrm{~A}$ and 548 are located on the upper continental slope. Several stratigraphic gaps were noted, especially in the Oligocene and Miocene.

Upper Paleocene to lower Eocene: Hole 548A, Samples 28-2, 61-62 cm to 23-1, 59-60 cm. Dominant characteristics are scarce carbonates and relatively abundant quartz $(12-15 \%)$. Clay minerals are mainly smectites $(80 \%)$; illites and undifferentiated kaolinites-chlorites are simply present.

Middle and upper Eocene: Hole 548A, Samples 22-2, $90-91 \mathrm{~cm}$ to $17-1,13-14 \mathrm{~cm}$. The sediments contain far more carbonate $(\sim 60-70 \%)$, and detrital quartz is scarce. Carbonates are always represented by normal calcite, except at the top of the Eocene, where occurrence of magnesian calcite has been noted (Sample 17-1, 13-14 cm). As in the lower Eocene, clay minerals are very rich in smectites; illites are always present, but kaolinites and chlorites are absent.

Oligocene: Hole 548A, Samples $16-2,49-50 \mathrm{~cm}$ to $15-1,32-33 \mathrm{~cm}$. Sediment is mostly carbonate (calcite); quartz is meager if not absent altogether. The clay phase for the most frequently occurring minerals remains similar to that in the Eocene, but kaolinites and chlorites reappear in limited quantities.

Lower and middle Miocene: Hole 548A, Samples $14-2,20-22 \mathrm{~cm}$ to $12-2,65-66 \mathrm{~cm}$. Deposits remain dominantly carbonate ( $70 \%$ normal calcite), except at the top of this interval, where there occurs a slight decrease in carbonate content. Clays continue to be mainly smectites; illites, kaolinites, and chlorites, more evident on the diffractograms, still occur in low proportions. Traces of zeolite (clinoptilolite) are present in the fraction slightly larger than $2 \mu \mathrm{m}$.

Upper Miocene: Hole 548A, Samples 11-2, 47-48 cm to $2-1,107-112 \mathrm{~cm}$. Carbonates are not as abundant as in the lower to middle Miocene $(\sim 40-50 \%)$, and detrital-quartz content sometimes exceeds $20 \%$. Clay fractions are less dominated by smectites than in the underlying deposits, and smectites tend to decrease progressively toward the top. Conversely, illite contents increase, whereas kaolinites and chlorites become more abundant than in the interval just below. The associated zeolite traces (clinoptilolite) are still evident in the very first upper Miocene levels.

Pliocene and Pleistocene: Hole 548, Samples 33-2, $80-81 \mathrm{~cm}$ to $1-1,1-2 \mathrm{~cm}$. The sediments contain less and less carbonate, whereas the bulk mineralogical composition oscillates considerably in the upper Pliocene and Pleistocene (from Core 19 to the top of the hole). From Core 15 to Sample 8-3, 90-91 cm, we noted the occurrence of magnesian calcite, aragonite, siderite, and anhydrite. Quartz is abundant $(30 \%)$. Clay minerals are mainly illites; smectites are always present, but in far less dominant proportions; kaolinites and chlorites abound. Numerous mineralogical changes occur in Core 15, with regard to both bulk sediments (carbonate, quartz, feldspars) and clay minerals. 
Site 549 (Fig. 3, Table 2)

This site is on the continental margin, where the Tertiary sediment record is well developed and complete, from Paleocene to the top of the Oligocene. Quaternary formations overlie the very condensed Miocene series. Indeed, the Pliocene has not been differentiated or recognized (see site chapter, this vol.).

Upper Paleocene: Hole 549, Samples 20-2, 63-65 cm to $16-1,51-52 \mathrm{~cm}$. This interval is marked by a calcite content of $\sim 50 \%$ and by low quartz contents. Clay minerals are mostly smectites, with some rare illites and undifferentiated kaolinite and chlorite traces.

Lower Eocene: Hole 549, Samples $15-2,51-53 \mathrm{~cm}$ to $10-1,44-46 \mathrm{~cm}$. This interval is richer in quartz $(10 \%)$ than the previous one; calcite contents remain unchanged. Clay fractions are always dominated by smectites, despite the slight smectite decrease in content; illite contents increase, whereas the occurrence of kaolinite and chlorite becomes more evident.

Middle and upper Eocene: Hole 549, Samples 9-2, $57-58 \mathrm{~cm}$ to $2-1,24-27 \mathrm{~cm}$ and Hole $549 \mathrm{~A}$, Samples $42-1,16-18 \mathrm{~cm}$ to $16-1,80-83 \mathrm{~cm}$. Carbonates (exclusively normal calcite) increase from the base to the top $(70-80 \%)$. Conversely, quartz gradually disappears. Smectites are dominant; illites, kaolinites, and chlorites are present. Within this episode the clay assemblage remains practically unchanged from below.

Oligocene: Hole 549A, Samples $15-2,20-23 \mathrm{~cm}$ to $6-1,90-91 \mathrm{~cm}$. Carbonate dominance resumes $(80 \%)$; quartz is scarce. The occurrence of siderite is to be noted from Sample 15-1, 20-23 cm to Sample 13-1, 20-23 cm; it corresponds to an increase of $\mathrm{Fe}$ in the acidic washing (see geochemical data, this chapter). Clay fractions are marked by a smectite decrease and a slight illite increase in comparison with the sediments below. Kaolinites and chlorites recur in limited quantities, after complete absence, from Sample 15-2, 20-23 cm to Sample 13-2, 20 $23 \mathrm{~cm}$.

Miocene: Hole 549A, Samples 5-2, 70-71 cm to 4-2, $80-81 \mathrm{~cm}$. This interval remains very rich in carbonate $(80 \%)$, with a slightly higher quartz content than in the Oligocene. Clay fractions are marked by an important decrease in smectite (70-50\%). Illites, kaolinites, and chlorites become progressively well represented.

Pleistocene: Hole 549A, Samples 3-5, $80-83 \mathrm{~cm}$ to $1-1,8-11 \mathrm{~cm}$. Directly overlying the Miocene, this inter$\mathrm{val}$ is characterized by clay minerals dominated mainly by illites; the smectite content falls to only $30 \%$, whereas kaolinites and chlorites are relatively abundant (about $15 \%$ each). As at Site 548, sudden variations in the proportions of different clay species are to be noted. Insufficient sample size prevented X-ray analysis of Pleistocene deposits.

\section{Site 550 (Fig. 4, Table 3)}

This site is on the abyssal plain. The Tertiary formations studied are the top of upper Paleocene, the lower Eocene, and the Miocene and Pliocene (no Oligocene level has been investigated).
Upper Paleocene: Hole 550, Samples 35-2, 61-62 cm to $34-4,61-62 \mathrm{~cm}$. Carbonates are poorly represented in Core 35 but more abundant $(40 \%)$ in Core 34 . In the fine fraction, smectite is practically the only mineral represented at the base of Core 35; in Core 34 it is accompanied by some illite, kaolinite, and chlorite.

Eocene: Hole 550, Samples 34-1, 61-62 to 25-1, $33-35 \mathrm{~cm}$. The carbonates in the lower Eocene sediments include 40 to $50 \%$ calcite. $\mathrm{Mg}$-calcite traces are apparent only in Sample 34-2, 61-62 cm. Quartz is relatively abundant $(10 \%)$. Clay minerals are mainly smectites, accompanied by some illite, kaolinite, and chlorite. A minor decrease of smectites occurs from base to the top of this interval, to the advantage of other clay minerals.

Lower middle Miocene: Hole 550, Samples 23-2, $68-70 \mathrm{~cm}$ to $17-2,22-26 \mathrm{~cm}$. This interval contains more carbonate than the Eocene, but undergoes considerable calcite variations; the percentage of quartz also fluctuates widely. Clay fractions are characterized by the dominance of smectite and illite; kaolinites and chlorites become progressively important. Zeolites (clinoptilolite) (Cores 23, 22, and 19) appear in the grain-size fraction slightly larger than $2 \mu \mathrm{m}$.

Upper Miocene: Hole 550, Samples 16-2, $34-38 \mathrm{~cm}$ to $10-1,31-34 \mathrm{~cm}$. This section contains very abundant carbonate (70-80\% calcite) and very little quartz. Clay minerals are mainly smectites at the bottom, progressively replaced by illites upsection.

Pliocene: Hole 550, Samples 9-2, 80-85 cm to 2-1, $43-46 \mathrm{~cm}$. Just as in the Miocene, the major mineralogical characteristic is the dominance of carbonates and a very low quartz content. Clay minerals are mainly illites, kaolinites, and chlorites. Smectites increase in the upper levels (Cores 3 and 2), which contain mixed Pliocene and upper Miocene faunas. This mixing could explain the relative smectite abundance, probably a result of reworking of the older formations.

\section{Summary of Variability}

\section{Lateral Variations}

Sediments from Site 548- the closest to the continental domain - are throughout the Cenozoic richer in detrital quartz than sediments from Sites 549 and 550, which are in deeper water. The lateral variation of the carbonate contents corresponds to relatively important variations in time: During the early Eocene and the Miocene-Piiocene, maximum carbonate accumulation rates, relative to other components, prevailed at the deeper sites; the opposite occurred in the middle/late Eocene and the Oligocene. Conversely, the different clay mineral assemblages never show, for any period, important lateral variations (Fig. 5).

\section{Vertical Variations}

The most general trend of the vertical mineralogical variation is, at all sites, an essentially regular decrease of the smectite/(illite + chlorite) ratio from the Paleocene to the Pleistocene. Although progressive, this vari- 


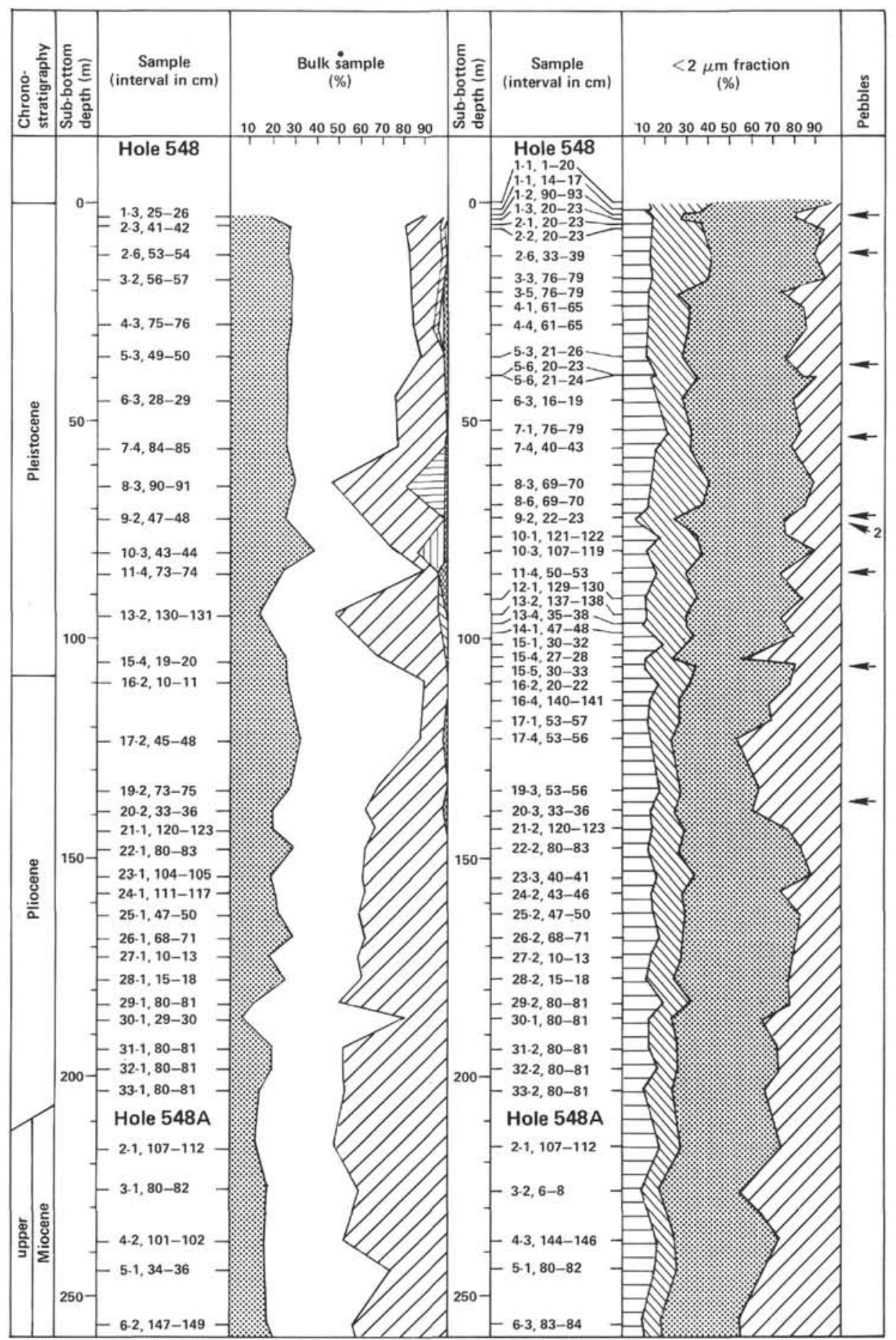

Figure 2. Mineralogical log, Site 548 (Holes 548 and 548A).

ation is marked by different stages which permit one to distinguish several clay mineralogical units (Fig. 5). Some of these stages or units coincide with changes of the nonclay components (see Figs. 2-4).

1. A first unit is composed of the Paleocene and/or lower Eocene intervals studied (Hole 548A: Samples 28-2, 61-62 cm to 23-1, 59-60 cm; Hole 549: Samples 20-2, 63-65 cm to $10-1,44-46 \mathrm{~cm}$; Hole 550: Samples $35-2,61-62 \mathrm{~cm}$ to $25-1,33-35 \mathrm{~cm})$. Smectites are practically the sole constituents of the clay fractions. Sedi- ments at Sites 549 and 550 have average carbonate contents and low quartz contents; those at Site 548 have low carbonate contents and relatively high quartz contents.

2. A second unit groups middle and upper Eocene, Oligocene, and lower to middle Miocene deposits (Hole 548A: Samples 22-1, 90-91 cm to 11-2, 47-48 cm; Hole 549: Samples 9-1, 36-37 cm to 6-1, 90-91 cm; Hole 550: Samples 23-2, 68-70 cm to $16-1,34-39 \mathrm{~cm}$ ).

In comparison with the first unit, the second is, on the whole, characterized by an increased carbonate con- 




Figure 2. (Continued).

tent and a decreased quartz content. This change is well marked in Hole 548A, where an abrupt increase of carbonates occurs between Samples 23-1, 59-60 cm and $22-1,90-91 \mathrm{~cm}$. In this hole, the decrease in quartz content may be partially the result of the dilution effect of carbonates. At other sites, the variation of quartz and carbonate contents is less clear.

As in the first unit, this one is marked by the predominance of smectite, with the smectite/illite ratio decreasing upward. Chlorite and kaolinite, present in small pro- portions, are in general poorly differentiated at Sites 548 and 549, but well differentiated at Site 550 .

3. A third unit is made up of upper Miocene and basal Pliocene deposits. The unit is well developed at Site 548 , relatively condensed at Site 550 , and very condensed at Site 549. The boundary with the second unit is abrupt in Hole 548A (between Samples 11-2, 47-48 cm and $10-3,72-73 \mathrm{~cm}$ ) and characterized by a sizable increase in quartz content and decrease in carbonate content. At all sites, the boundary is marked by a rapid de- 


\section{G. CHENNAUX ET AL.}

Table 1. Mineralogical analyses (\%), Site 548 (Holes 548 and 548A).

\begin{tabular}{|c|c|c|c|c|c|c|c|c|c|c|c|c|c|}
\hline \multirow[b]{2}{*}{$\begin{array}{c}\text { Sample } \\
\text { (interval in cm) }\end{array}$} & \multirow{2}{*}{$\begin{array}{l}\text { Sub-bottom } \\
\text { depth } \\
\text { (m) }\end{array}$} & \multicolumn{7}{|c|}{ Bulk sediment } & \multicolumn{5}{|c|}{$<2 \mu \mathrm{m}$ fraction } \\
\hline & & Calcite & Dolomite & Siderite & $\begin{array}{l}\text { Arag- } \\
\text { onite }\end{array}$ & $\begin{array}{c}\mathrm{Mg} \\
\text { calcite }\end{array}$ & Quartz & $\begin{array}{l}\text { Anhy- } \\
\text { drite }\end{array}$ & $\begin{array}{c}\text { Smec- } \\
\text { tite }\end{array}$ & Illite & $\begin{array}{l}\text { Kao- } \\
\text { linite }\end{array}$ & Chlorite & $\begin{array}{c}\text { Kaolinite + } \\
\text { chlorite }\end{array}$ \\
\hline \multicolumn{14}{|l|}{ Hole 548} \\
\hline $1-1,0-2$ & 0.10 & & & & & & & & 5 & 53 & 14 & 28 & \\
\hline $1-1,14-17$ & 1.50 & & & & & & & & 11 & 52 & 15 & 22 & \\
\hline $1-2,90-93$ & 2.42 & & & & & & & & 19 & 52 & 13 & 16 & \\
\hline $1-3,20-23$ & & & & & & & & & 20 & 52 & 14 & 14 & \\
\hline $1-3,25-26$ & 3.20 & 8 & 1 & & & & 18 & & & & & & \\
\hline $2-1,20-23$ & 4.20 & & & & & & & & 14 & 49 & 16 & 21 & \\
\hline $2-2,20-23$ & 5.70 & & & & & & & & 7 & 55 & 15 & 23 & \\
\hline $2-3,41-42$ & 7.40 & 16 & 2 & & & & 28 & 1 & & & & & \\
\hline $2-6,33-39$ & & & & & & & & & 11 & 47 & 14 & 28 & \\
\hline $2-6,53-54$ & 11.83 & 15 & 2 & & & & 27 & & & & & & \\
\hline $3-2,56-57$ & 16.56 & 14 & 2 & & & & 29 & 1 & & & & & \\
\hline $3-3,76-79$ & 17.26 & & & & & & & & 6 & 53 & 16 & 25 & \\
\hline $3-5,76-79$ & 20.26 & & & & & & & & 25 & 49 & 14 & 12 & \\
\hline $4-1,61-65$ & 23.61 & & & & & & & & 16 & 52 & 14 & 18 & \\
\hline $4-3,75-76$ & 26.75 & 10 & 1 & & & 4 & 28 & 1 & & & & & \\
\hline $4-4,61-65$ & 28.11 & & & & & & & & 14 & 54 & 13 & 19 & \\
\hline $4-6,20-23$ & 30.70 & & & & & & & & 16 & 50 & 17 & 17 & \\
\hline $5-3,21-26$ & & & & & & & & & 24 & 48 & 12 & 16 & \\
\hline $5-3,49-50$ & 35.21 & 10 & 1 & & & & 26 & 1 & & & & & \\
\hline $5-6,21-24$ & 39.11 & & & & & & & & 9 & 55 & 14 & 22 & \\
\hline $6-3,16-19$ & 45.16 & & & & & & & & 22 & 50 & 17 & 11 & \\
\hline $6-3,28-29$ & 45.16 & 24 & & & & & 27 & 1 & & & & & \\
\hline $7-1,76-79$ & 52.26 & & & & & & & & 17 & 50 & 22 & 11 & \\
\hline $7-4,40-43$ & 56.40 & & & & & & & & 21 & 47 & 16 & 16 & \\
\hline $7-4,84-85$ & 56.40 & 23 & & & & & 26 & & & & & & \\
\hline $8-3,69-70$ & & & & & & & & & 11 & 48 & 14 & 27 & \\
\hline $8-3,90-91$ & 64.69 & 37 & 1 & 1 & 16 & & 30 & & & & & & \\
\hline $8-6,69-70$ & 69.19 & & & & & & & & 15 & 47 & 13 & 25 & \\
\hline $9-2,22-23$ & & & & & & & & & 25 & 51 & 7 & 17 & \\
\hline $9-2,47-48$ & 72.22 & 40 & & 1 & & & 26 & & & & & & \\
\hline $10-1,121-122$ & $76-21$ & & & & & & & & 24 & 40 & 18 & 18 & \\
\hline $10-3,43-44$ & 78.43 & 12 & & & & 13 & & & & & & & \\
\hline $10-3,101-119$ & 78.43 & & & & & & & & 11 & 51 & 12 & 26 & \\
\hline $11-4,50-53$ & & & & & & & & & 27 & 43 & 17 & 13 & \\
\hline $11-4,73-74$ & 85.00 & 7 & & & & & 23 & 3 & & & & & \\
\hline $12-1,129-130$ & 90.79 & & & & & & & & 16 & 48 & 12 & 24 & \\
\hline $13-2,130-131\}$ & & 48 & & 4 & & & 14 & & & 80 & . & 2 & \\
\hline $13-2,137-138$ & 94.87 & & & & & & & & 27 & 43 & 12 & 18 & \\
\hline $13-4,35-38$ & 96.85 & & & & & & & & 23 & 47 & 10 & 20 & \\
\hline $14-1,47$ & 99.47 & & & & & & & & 19 & 47 & 17 & 17 & \\
\hline $15-2,30-32$ & 101.80 & & & & & & & & 30 & 41 & 20 & 9 & \\
\hline $15-4,19-20$ & 104.69 & 31 & & & 1 & & 26 & & & & & & \\
\hline $15-4,27-28$ & 104.69 & & & & & & & & 42 & 34 & 12 & 12 & \\
\hline $15-5,30-33$ & 106.30 & & & & & & & & 19 & 46 & 11 & 24 & \\
\hline $16-2,10-11$ & 11010 & 10 & & & & & 27 & & & & & & \\
\hline $16-2,20-22$ & 110.10 & & & & & & & & 22 & 46 & 18 & 14 & \\
\hline $16-4,140-141$ & 114.40 & & & & & & & & 32 & 42 & 13 & 13 & \\
\hline $17-1,53-57$ & 118.53 & & & & & & & & 30 & 43 & 12 & 15 & \\
\hline $17-2,45-48$ & 119.95 & 12 & & & & & 33 & 1 & & & & & \\
\hline $17-4,53-56$ & 123.03 & & & & & & & & 47 & 30 & 14 & 9 & \\
\hline $19-2,73-75$ & 133.23 & 33 & & & & & 28 & & & & & & \\
\hline $19-3,53-56$ & 134.53 & & & & & & & & 36 & 36 & 18 & 10 & \\
\hline $20-2,33-36$ & 137.83 & 37 & & 1 & & & 20 & & & & & & \\
\hline $20-3,33-36$ & 139.33 & & & & & & & & 39 & 36 & 14 & 11 & \\
\hline $21-1,20-23$ & 141.20 & 33 & & & & & 20 & & & & & & \\
\hline $21-2,120-123$ & 143.70 & & & & & & & & 23 & 47 & 15 & 15 & \\
\hline $22-1,80-83$ & 146.80 & 38 & & & & & 30 & & & & & & \\
\hline $22-2,80-83$ & 148.30 & & & & & & & & 17 & 57 & 13 & 13 & \\
\hline $23-1,104-105$ & 152.04 & 39 & & & & & 20 & & & & & & \\
\hline $23-3,40-41$ & 154.40 & & & & & & & & 12 & 54 & 17 & 17 & \\
\hline $24-1,114-117$ & 157.14 & 38 & & & & & 22 & & & & & & \\
\hline $24-2,43-46$ & 157.93 & & & & & & & & 26 & 46 & 14 & 14 & \\
\hline $25-1,47-50$ & 161.45 & 41 & & & & & 23 & & & & & & \\
\hline $25-2,47-50$ & 162.97 & & & & & & & & 18 & 52 & 15 & 15 & \\
\hline $26-1,68-71$ & 166.68 & 38 & & & & & 30 & & & & & & \\
\hline $26-2,68-71$ & 168.18 & & & & & & & & 20 & 51 & 18 & 11 & \\
\hline $27-1,10-13$ & 171.10 & 42 & & & & & 19 & & & & & & \\
\hline $27-2,10-13$ & 172.60 & & & & & & & & 22 & 15 & 14 & 14 & \\
\hline $28-1,15-18$ & 176.15 & 39 & & & & & 26 & & & & & & \\
\hline $28-2,15-18$ & 177.65 & & & & & & & & 24 & 52 & 12 & 12 & \\
\hline
\end{tabular}


Table 1. (Continued).

\begin{tabular}{|c|c|c|c|c|c|c|c|c|c|c|c|c|c|}
\hline \multirow[b]{2}{*}{$\begin{array}{c}\text { Sample } \\
\text { (interval in } \mathrm{cm} \text { ) }\end{array}$} & \multirow{2}{*}{$\begin{array}{l}\text { Sub-bottom } \\
\text { depth } \\
\text { (m) }\end{array}$} & \multicolumn{7}{|c|}{ Bulk sediment } & \multicolumn{5}{|c|}{$<2 \mu \mathrm{m}$ fraction } \\
\hline & & Calcite & Dolomite & Siderite & $\begin{array}{l}\text { Arag- } \\
\text { onite }\end{array}$ & $\begin{array}{c}\mathrm{Mg} \\
\text { calcite }\end{array}$ & Quartz & $\begin{array}{l}\text { Anhy- } \\
\text { drite }\end{array}$ & $\begin{array}{c}\text { Smec- } \\
\text { tite }\end{array}$ & Illite & $\begin{array}{l}\text { Kao- } \\
\text { linite }\end{array}$ & Chlorite & $\begin{array}{c}\text { Kaolinite + } \\
\text { chlorite }\end{array}$ \\
\hline \multicolumn{14}{|l|}{ Hole 548 (Cont.) } \\
\hline $29-1,80-81$ & 181.80 & 50 & & & & & 12 & & & & & & \\
\hline $29-2,80-81$ & 183.30 & & & & & & & & 22 & 46 & 20 & 12 & \\
\hline $30-1,29-30$ & & 19 & & & & & 6 & & & & & & \\
\hline $30-1,80-81\}$ & 186.29 & & & & & & & & 35 & 42 & 13 & 10 & \\
\hline $31-1,80-81$ & 191.80 & 48 & & & & & 20 & & & & & & \\
\hline $31-2,80-81$ & 193.30 & & & & & & & & 28 & 46 & 13 & 13 & \\
\hline $32-1,80-81$ & 196.80 & 48 & & & & & 20 & & & & & & \\
\hline $32-2,80-81$ & 198.30 & & & & & & & & 27 & 47 & 17 & 9 & \\
\hline $33-1,80-81$ & 201.80 & 47 & & & & & 14 & & & & & & \\
\hline $33-2,80-81$ & 203.30 & & & & & & & & 34 & 43 & 10 & 13 & \\
\hline \multicolumn{14}{|l|}{ Hole $548 \mathrm{~A}$} \\
\hline $2-1,107-112$ & 216.07 & 53 & & & & & 12 & & 26 & 47 & 18 & 9 & \\
\hline $3-1,80-82$ & 225.30 & 41 & & & & & 18 & & & & & & \\
\hline $3-2,6-8$ & 226.06 & & & & & & & & 46 & 37 & 9 & 8 & \\
\hline $4-2,101-102$ & 235.51 & 48 & & & & & 15 & & & & & & \\
\hline $4-3,144-146$ & 237.44 & & & & & & & & 28 & 48 & 16 & 8 & \\
\hline $5-1,34-36$ & 243.84 & 26 & & & & & 17 & & & & & & \\
\hline $5-1,80-82$ & 244.30 & & & & & & & & 34 & 41 & 15 & 10 & \\
\hline $6-2,147-149$ & 255.97 & 43 & & & & & 18 & & & & & & \\
\hline $6-3,83-84$ & 256.83 & & & & & & & & 46 & 36 & 9 & 9 & \\
\hline $7-1,121-123$ & 262.71 & 38 & & & & & 28 & & & & & & \\
\hline $7-2,94-96$ & 267.44 & & & & & & & & 45 & 35 & 13 & 7 & \\
\hline $8-1,65-66$ & 272.65 & 46 & & & & & 22 & & & & & & \\
\hline $8-2,106-107$ & 274.56 & & & & & & & & 52 & 34 & 7 & 7 & \\
\hline $9-1,78-79$ & 282.28 & 36 & & & & & 19 & & & & & & \\
\hline $9-2,78-79$ & 283.78 & & & & & & & & 48 & 32 & 12 & 8 & \\
\hline $10-2,72-73$ & 293.22 & 34 & & & & & 28 & & & & & & \\
\hline $10-3,72-73$ & 294.72 & & & & & & & & 32 & 44 & 12 & 12 & \\
\hline $11-1,47-48$ & 300.97 & 47 & & & & & 16 & & & & & & \\
\hline $11-2,47-48$ & 302.47 & & & & & & & & 59 & 27 & 7 & 7 & \\
\hline $12-1,65-66$ & 310.65 & 64 & & & & & 3 & & & & & & \\
\hline $12-2,65-66$ & 312.15 & & & & & & & & 57 & 33 & 5 & 5 & \\
\hline $13-1,71-72$ & 320.50 & 15 & & & & & 8 & & & & & & \\
\hline $13-1,100-101\}$ & 320.50 & & & & & & & & 57 & 29 & 9 & 5 & \\
\hline $14-1,20-22$ & 329.20 & 72 & & & & & 3 & & & & & & \\
\hline $14-2,20-22$ & 330.70 & & & & & & & & 62 & 32 & 3 & 3 & \\
\hline $15-1,32-33$ & 338.82 & 71 & & & & & 1 & & & & & & \\
\hline $15-3,32-33$ & 341.82 & & & & & & & & 66 & 34 & $\mathrm{~T}$ & $\mathrm{~T}$ & \\
\hline $16-1,49-50$ & 348.49 & 74 & & & & & 1 & & & & & & \\
\hline $16-2,49-50$ & 349.99 & & & & & & & & 82 & 16 & & & 2 \\
\hline $17-1,13-14$ & 357.63 & 50 & & & & 16 & 3 & & & & & & \\
\hline $17-1,69-72$ & 358.19 & & & & & & & & 75 & 20 & & & 5 \\
\hline $18-1,40-41$ & 367.41 & 65 & & & & & 3 & & & & & & \\
\hline $18-2,40-41$ & 368.90 & & & & & & & & 80 & 20 & & & \\
\hline $19-1,51-53$ & 377.01 & 65 & & & & & 3 & & & & & & \\
\hline $19-1,113-115$ & 377.63 & & & & & & & & 85 & 13 & & & 2 \\
\hline $20-1,84-85$ & 386.84 & 59 & & & & & 4 & & & & & & \\
\hline $20-2,49-50$ & 387.99 & & & & & & & & 82 & 18 & & & \\
\hline $21-1,71-73$ & 396.21 & 58 & & & & & 6 & & & & & & \\
\hline $21-2,71-72$ & 397.71 & & & & & & & & 85 & 15 & & & \\
\hline $22-1,90-91$ & 405.90 & 60 & & & & & 6 & & & & & & \\
\hline $22-2,90-91$ & 407.40 & & & & & & & & 87 & 13 & & & \\
\hline $23-1,59-60$ & 415.19 & 16 & & & & & 13 & 1 & & & & & \\
\hline $23-2,58-59$ & 416.58 & & & & & & & & 66 & 19 & & & 15 \\
\hline $24-1,41-42$ & 424.41 & 16 & & & & & 12 & & & & & & \\
\hline $24-2,14-15$ & 425.64 & & & & & & & & 83 & 11 & & & 6 \\
\hline $25-1,31-32$ & 433.81 & 12 & & & & & 10 & & & & & & \\
\hline $25-2,81-82$ & 435.81 & & & & & & & & 76 & 14 & & & 10 \\
\hline $27-1,59-60$ & 453.09 & 37 & & & & & 10 & & & & & & \\
\hline $27-2,61-62$ & 454.61 & & & & & & & & 82 & 12 & & & 6 \\
\hline $28-1,61-62$ & 462.61 & 26 & & & & & 15 & & & & & & \\
\hline $28-2,61-62$ & 464.11 & & & & & & & & 77 & 15 & & & 8 \\
\hline
\end{tabular}




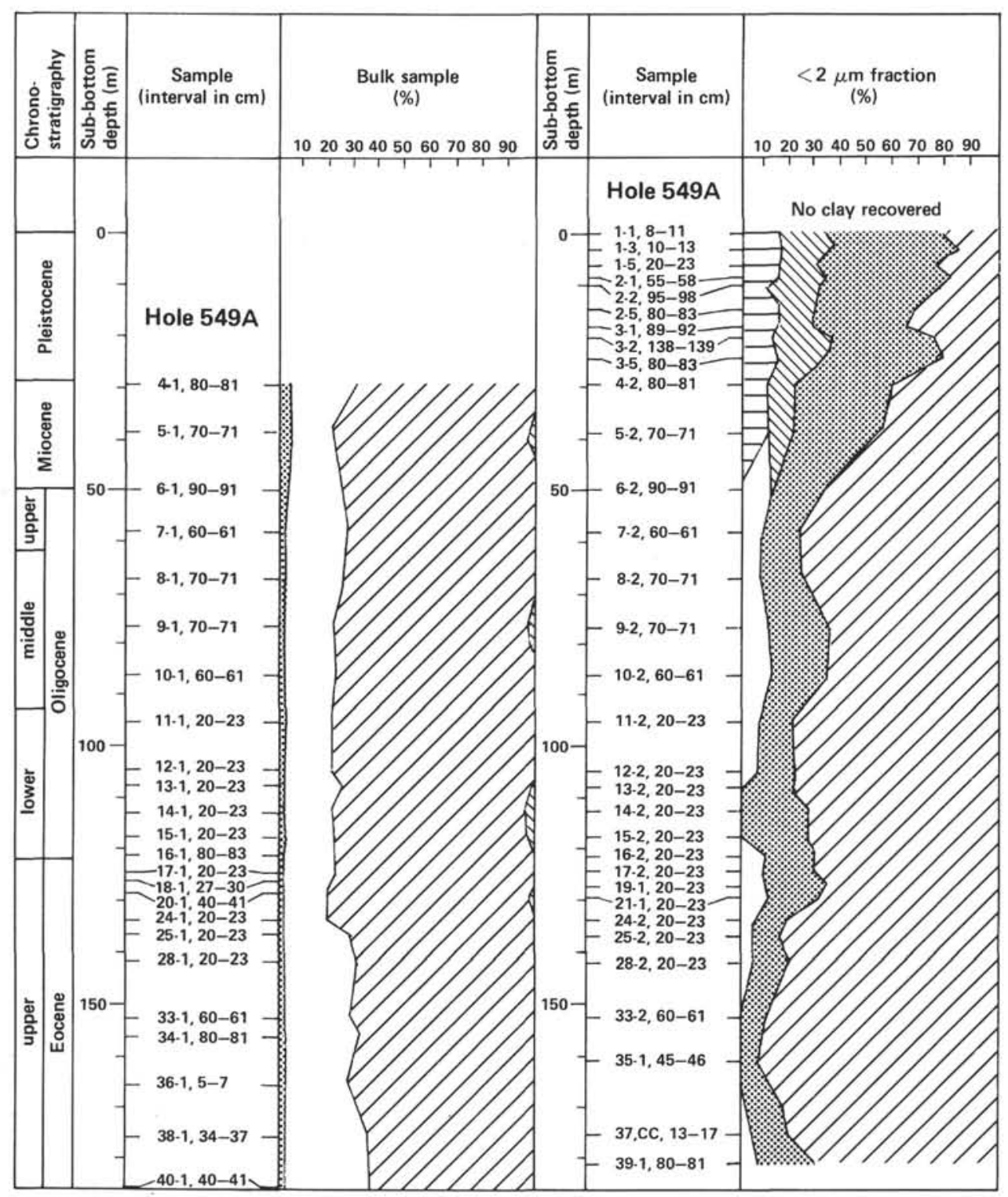

Figure 3. Mineralogical log, Site 549 (Holes 549A and 549). Legend as in Fig. 2. No pebbles were recovered from the part of the section shown.

crease of the smectite/illite ratio. This change is clear in Hole 548A (between Samples 11-2, 47-48 cm and 10-2, $72-73 \mathrm{~cm}$ ), in Hole 549 (between Samples 6-2, 90-91 cm and 5-2, 70-71 cm), and in Hole 550 (between Samples $16-2,34-38 \mathrm{~cm}$ and $15-1,35-39 \mathrm{~cm}$ ).

4. A thin fourth unit is characterized by the recurrence of smectites. It appears in the median part of the Pliocene in Hole 548 (Samples 20-3, 33-36 cm to 17-4, 53-56 cm) and Hole 550 (Samples 4-2, $44-48 \mathrm{~cm}$ to 3-2, $51-54 \mathrm{~cm}$ ). This unit does not exist in Hole 549A, where the Pliocene is missing. This fourth unit could be associated with the reworking of underlying deposits and thus explain the increase in content of smectite. Such redeposition has been suspected at Site 550 (see site chapter, this vol.).

5. A fifth unit is made up of uppermost Pliocene and Quaternary deposits. This unit was the object of a complete mineralogical study only in the bulk sediments from Hole 548 (Samples $17-1,53-57 \mathrm{~cm}$ to $1-1,1-2 \mathrm{~cm}$ ). Only clay minerals were studied in Hole 549A (Samples $5-2,70-71 \mathrm{~cm}$ to $1-1,8-11 \mathrm{~cm})$. The unit is missing at Site 550. Its prominent characteristic is the strong predominance of illite, the relative abundance of chlorite, and the great variability in mineralogical composition. In Hole 548, where this unit is well developed, one notes a relatively high abundance of quartz and an irregular carbonate representation, within which occur dolomite, $\mathrm{Mg}$ calcites, and aragonite.

\section{Interpretation of Leg 80 Mineralogical Data}

Since the Millot studies (1964), marine clays have often been considered as deriving more from continental erosion than from authigenic and diagenetic processes in the oceanic environment itself. In the Atlantic Ocean in particular, detrition of lands and soils clearly appears as the dominant mechanism responsible for clay-miner- 


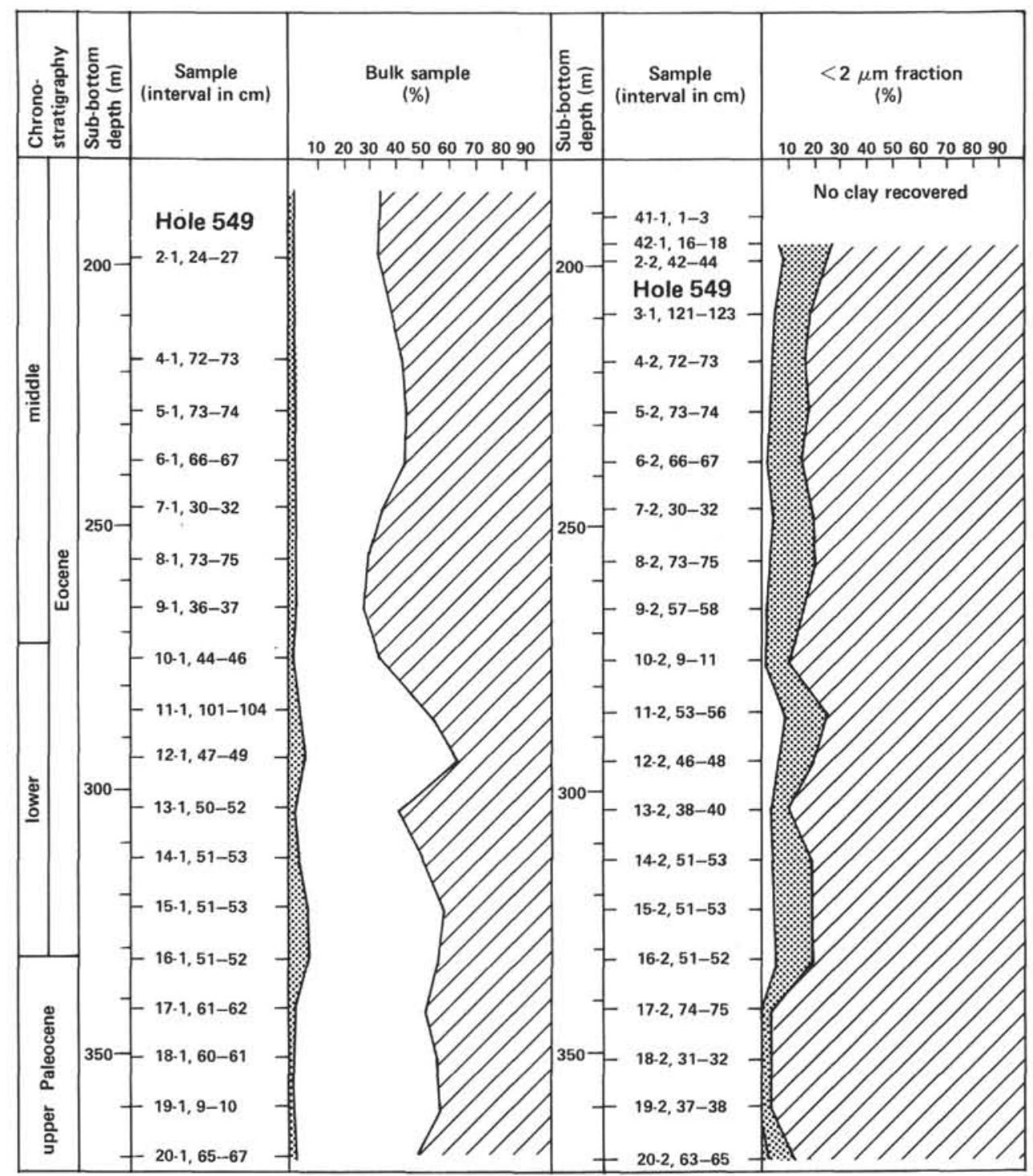

Figure 3. (Continued).

al supply, both for modern deposits (Biscaye, 1965; Griffin et al., 1968; Rateev et al., 1969; Grousset et al., in press) and for Mesozoic and Cenozoic continental margin sediments (Courtois and Chamley, 1978; Chamley, 1979). So, leaving aside localized strata in which a particular frequent occurrence of volcanic glasses could contribute to the in situ genesis of bentonitic clays (Knox, this volume), we discuss now the significance of Leg 80 clay minerals, keeping always in mind the major importance of the continental detrital supply.

The abundance of smectites from the Paleocene to the middle Miocene should suggest an inheritance from the clay fractions of soils developed under a hot climate with contrasting seasons similar to those described by Paquet (1969) in present-day badly drained areas of Africa. The genesis of these clays on the continent could also be favored by the presence of volcanic basic parent rocks. The record of this hot climate of alternating seasons has already been recognized at the Tertiary base within various zones of the northeast Atlantic province (Latouche and Maillet, 1978, 1979): at Rockall (Leg 48) in the Eocene (Oligocene absent) and off Portugal (Leg $47)$ in the Eocene and Oligocene.

The additional presence of illite and chlorite-rather unresistant minerals, considered as "primary"-shows, however, that supply from alterations rich in smectites is not exclusive. These minerals figure at the same time as smectites in the deposits of the Bay of Biscay (Latouche and Maillet, 1980), and west of Portugal (Chamley et al., 1979). In these areas, primary minerals can be attributed to the consecutive erosion of the Pyrenees. On Goban Spur, on the contrary, the presence of illite (and poorly crystallized chlorite) in the Paleogene cannot be linked to a tectonic rejuvenation of the neighboring continent, for marine deposits are essentially fine, calcareous, and clayey and scarcely quartziferous even at Site 548 , which is the drill site closest to the continent.

In the middle Miocene, major changes in the mineralogical composition of the deposit must be noted:

1. An important increase of the ratio (illite + chlorite)/smectites at all sites (in Hole 548A between Samples $11-2,47-48 \mathrm{~cm}$ and $10-3,72-73 \mathrm{~cm}$; in Hole 549A 
Table 2. Mineralogical analyses (\%), Site 549 (Holes 549A and 549).

\begin{tabular}{|c|c|c|c|c|c|c|c|}
\hline & Sub-bottom & & alk sedim & & & & $<2 \mu \mathrm{m}$ \\
\hline $\begin{array}{c}\text { Sample } \\
\text { (interval in } \mathrm{cm} \text { ) }\end{array}$ & $\begin{array}{l}\text { depth } \\
\text { (m) }\end{array}$ & Calcite & Siderite & Quartz & $\begin{array}{l}\text { Smec- } \\
\text { tite }\end{array}$ & Illite & $\begin{array}{l}\text { Kao- } \\
\text { linite }\end{array}$ \\
\hline Hole 549A & & & & & & & \\
\hline $1-1,8-11$ & 0.80 & & & & 23 & 46 & 14 \\
\hline $1-3,10-13$ & 3.20 & & & & 16 & 49 & is \\
\hline $1-5,20-23$ & 6.20 & & & & 26 & 46 & 14 \\
\hline $2-1,55-58$ & 8.55 & & & & 18 & 50 & 14 \\
\hline $2-2,95-98$ & 10.45 & & & & 21 & 49 & 10 \\
\hline $2-5,80-83$ & 14.80 & & & & 33 & 39 & 14 \\
\hline $3-1,89-92$ & 18.39 & & & & 36 & 36 & 14 \\
\hline 3-2, 138-139 & 20.38 & & & & 24 & 40 & 12 \\
\hline $\begin{array}{l}3-5,80-83 \\
4-1,80-81\end{array}$ & $\begin{array}{l}24.30 \\
27.80\end{array}$ & & & & 21 & 46 & 15 \\
\hline $\begin{array}{l}\begin{array}{r}4-1,80-81 \\
4-2,80-81\end{array} \\
4\end{array}$ & $\begin{array}{l}27.80 \\
29.30\end{array}$ & 70 & & 4 & & & \\
\hline 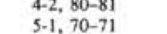 & $\begin{array}{l}29.30 \\
37.20\end{array}$ & 78 & 2 & 4 & 42 & 38 & 10 \\
\hline $5-2,70-71$ & 38.70 & & & & 46 & 34 & 10 \\
\hline $6-1,90-91$ & 46.90 & 76 & & 2 & & & \\
\hline $6-2,90-91$ & 48.40 & & & & 68 & 21 & \\
\hline $7-1,60-61$ & 56.10 & 73 & & & & & \\
\hline $7-2,60-61$ & $\begin{array}{l}57.60 \\
6 \leq 70\end{array}$ & & & & 77 & 15 & \\
\hline $8-1,70-71$ & 65.70 & 75 & & 2 & & & \\
\hline $8-2,70-71$ & 67.20 & & & & 76 & 17 & \\
\hline $\begin{array}{l}9-1,70-71 \\
9-2,70-71\end{array}$ & $\begin{array}{l}75.20 \\
76.70\end{array}$ & 76 & 3 & 1 & 66 & 23 & \\
\hline $10-1,60-61$ & 84.60 & 77 & & 1 & 100 & 23 & \\
\hline $10-2,60-61$ & 86.10 & 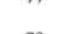 & & 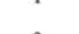 & 66 & 22 & \\
\hline $11-1,20-23$ & 93.70 & 79 & & 2 & & & \\
\hline $11-2,20-23$ & 95.20 & 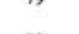 & & 2 & 81 & 13 & \\
\hline $12-1,20-23$ & 103.20 & 79 & & 1 & & & \\
\hline $12-2,20-23$ & 104.70 & & & & 79 & 14 & \\
\hline $13-1,20-23$ & 106.20 & 73 & 2 & 1 & e &  & \\
\hline $13-2,20-23$ & 107,70 & & & & 81 & 19 & \\
\hline $14-1,20-23$ & 111.20 & 76 & 4 & 1 & & & \\
\hline $14-2,20-23$ & 112.17 & & & & 73 & 27 & \\
\hline $15-1,20-23$ & 116.20 & 75 & 3 & 2 & & 6 & \\
\hline $\begin{array}{l}15-2,20-23 \\
16-1,80-83\end{array}$ & 120.30 & 78 & & 1 & 74 & 26 & \\
\hline $16-2,20-23$ & 121.80 & & & & 71 & 19 & \\
\hline $17-1,20-23$ & 122.70 & & 1 & & & , & \\
\hline $17-2,20-23$ & 124.20 & & & & 72 & 19 & \\
\hline $18-1,27-30$ & 125.77 & 78 & & 1 & 16 & 18 & \\
\hline $19-1,20-23$ & 127.20 & 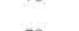 & & & 67 & 22 & \\
\hline $20-1,40-41$ & 128.40 & 79 & 2 & 1 & & & \\
\hline $21-1,20-23$ & 129.20 & & 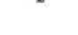 & 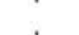 & 69 & 20 & \\
\hline $24-1,20-23$ & 132.20 & 81 & & 1 & & & \\
\hline $24-2,20-23$ & $\begin{array}{l}133.70 \\
135.20\end{array}$ & & & & 83 & 12 & \\
\hline $\begin{array}{l}25-1,20-23 \\
25-2,20-23\end{array}$ & $\begin{array}{l}135.20 \\
136.70\end{array}$ & 71 & & 1 & 85 & 10 & \\
\hline $28-1,20-23$ & $\begin{array}{l}130.20 \\
140.20\end{array}$ & 69 & & 1 & & & \\
\hline $28 \cdot 2,20-23$ & 141.70 & & & & 81 & 14 & \\
\hline $33-1,60-61$ & 151.10 & 72 & & $i$ & & & \\
\hline $33-2,60-61$ & 152.60 & & & & 90 & 10 & \\
\hline $34-1,80-81$ & 156.30 & 68 & & 8 & & & \\
\hline $35-1,45-46$ & 160.95 & & & & 93 & 7 & \\
\hline $36-1,5-7$ & $\begin{array}{l}165.55 \\
175.43\end{array}$ & 73 & & 1 & & & \\
\hline $37, \mathrm{CC}(13-17)$ & 175.43 & & & & 81 & 15 & \\
\hline $38-1,34-37$ & 175.84 & 66 & & 2 & & & \\
\hline $\begin{array}{l}39-1,80-81 \\
40,-40-4\end{array}$ & $\begin{array}{l}181.30 \\
185.90\end{array}$ & 64 & & 2 & 71 & 21 & \\
\hline $\begin{array}{l}40-1,40-41 \\
41-1,1-3\end{array}$ & $\begin{array}{l}\begin{array}{l}185.90 \\
190.51\end{array}\end{array}$ & 64 & & 2 & & & \\
\hline $42-1,16-18$ & 195.66 & & & & 74 & 19 & \\
\hline Hole 549 & & & & & & & \\
\hline $2-1,24-27$ & 198.74 & 65 & & 2 & & & \\
\hline $2-2,42-44$ & 200.42 & & & & 75 & 16 & \\
\hline $3-1,121-123$ & 209.21 & & & & 81 & 13 & \\
\hline $4-1,72-73$ & 216.28 & 56 & & 8 & & & \\
\hline $4-2,72-73$ & $\begin{array}{l}219.72 \\
2777\end{array}$ & & & & 83 & II & \\
\hline $5-1,73-74$ & $\begin{array}{l}227.73 \\
220\end{array}$ & 54 & & 3 & & & \\
\hline $\begin{array}{l}5-2,73-74 \\
6-1,66-67\end{array}$ & $\begin{array}{l}229.23 \\
223716\end{array}$ & $\$$ & & 3 & 82 & 13 & \\
\hline $\begin{array}{l}6-1,66-67 \\
6-2,66-67\end{array}$ & 238.66 & 32 & & & 85 & 13 & \\
\hline $7-1,30-$ & 246.30 & 64 & & 4 & & & \\
\hline $7-2,30$ & 247.80 & & & & 80 & 14 & \\
\hline $8-1,73-$ & 256.23 & 69 & & 4 & & & \\
\hline $8-2,73-$ & 257.73 & 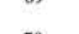 & & 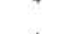 & 79 & 16 & \\
\hline $9-1,36-3$ & 265.36 & 70 & & 4 & & & \\
\hline $9-2,57-58$ & 268.10 & & & & 84 & 14 & \\
\hline $10-1,44-46$ & 274.94 & 64 & & 2 & & & \\
\hline 9 & 276.09 & & & & 90 & 8 & \\
\hline $11-1,101-104$ & $\begin{array}{l}285.01 \\
28603\end{array}$ & 45 & & 5 & & & \\
\hline $\begin{array}{l}11-2,53-56 \\
12-1,47-49\end{array}$ & $\begin{array}{l}286.03 \\
293.97\end{array}$ & 34 & & 7 & 75 & 13 & \\
\hline $12-2,46-48$ & 295.46 & 34 & & & 79 & 13 & \\
\hline $13-1,50-52$ & 303.50 & 57 & & 3 & & & \\
\hline $13 \cdot 2,38-40$ & 304.88 & & & & 89 & 7 & \\
\hline $14-1,51-53$ & 313.01 & 47 & & 5 & & & \\
\hline $14-2,51-53$ & 314.51 & & & & 80 & 14 & \\
\hline $15-1,51-53$ & 322.51 & 40 & & 9 & & & \\
\hline $15-2,51-$ & 324.01 & & & & 81 & 12 & \\
\hline si- & 332.01 & 42 & & 9 & & & \\
\hline 51 & 333 & & & & 80 & 12 & \\
\hline .61 & 341 & 47 & & 3 & & & \\
\hline $17-2,76-75$ & $\begin{array}{l}343.24 \\
351.0\end{array}$ & & & & 97 & 3 & \\
\hline $\begin{array}{l}18-1,60-61 \\
18-2,31-32\end{array}$ & $\begin{array}{l}351.10 \\
352.31\end{array}$ & 42 & & 2 & 97 & 3 & \\
\hline $\begin{array}{l}18-2,31-32 \\
19-1,9-10\end{array}$ & $\begin{array}{l}352.31 \\
360.09\end{array}$ & 41 & & 2 & (1, & 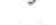 & \\
\hline $19-2,63-65$ & 362.13 & 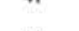 & & 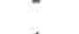 & 96 & 4 & \\
\hline $20-1,65-67$ & 370.15 & 51 & & 4 & 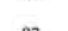 & & \\
\hline $20-2,63-65$ & 371.63 & & & & 87 & 10 & \\
\hline
\end{tabular}

Note: Bulk sediment contained no dolomite, aragonite, or anhydrite. 


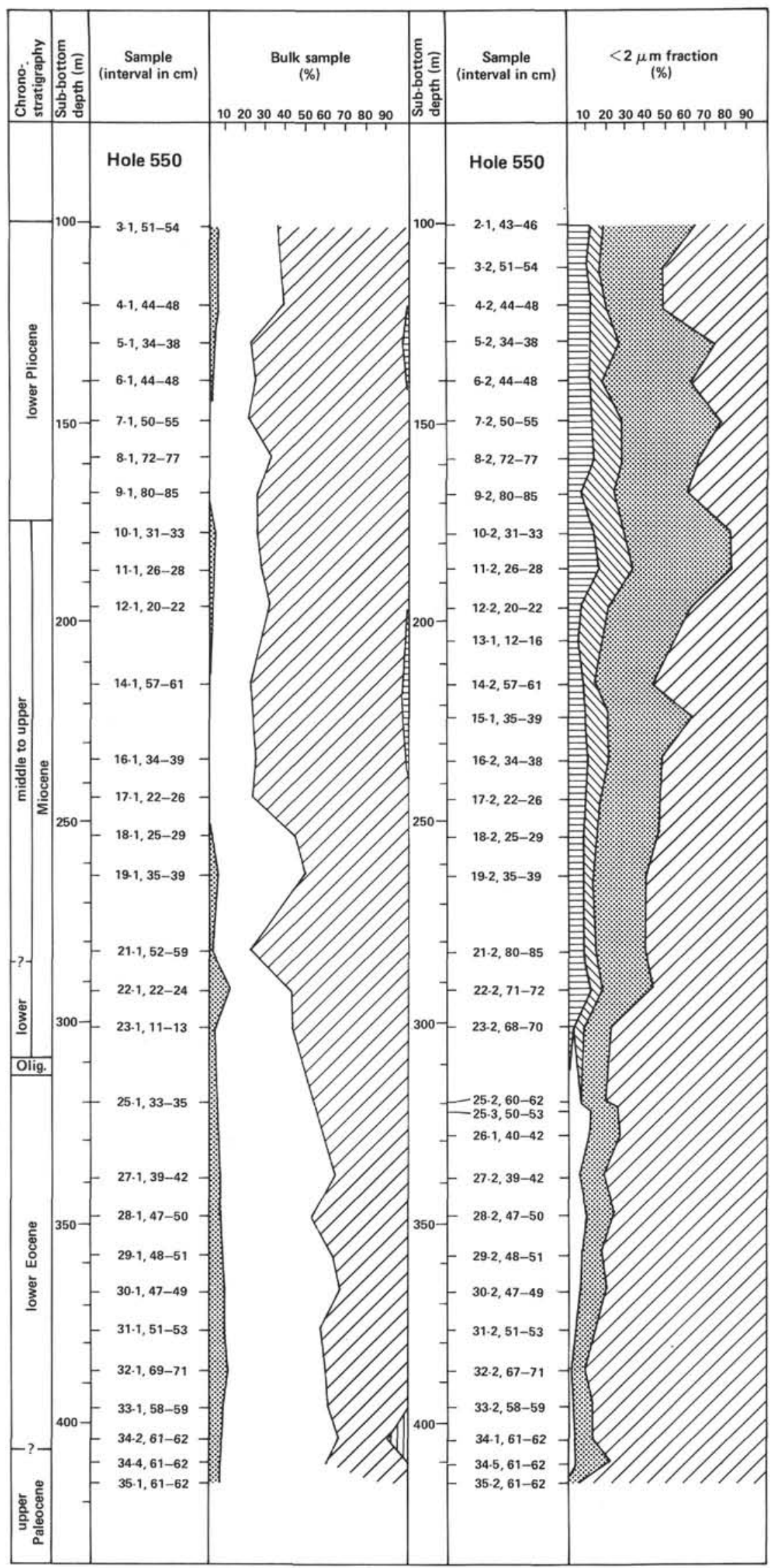

Figure 4. Mineralogical log, Site 550 (Hole 550). Legend as in Fig. 2. No pebbles were recovered from the part of the section shown. 
Table 3. Mineralogical analyses (\%), Site 550 (Hole 550).

\begin{tabular}{|c|c|c|c|c|c|c|c|c|c|c|}
\hline \multirow[b]{2}{*}{$\begin{array}{c}\text { Sample } \\
\text { (interval in } \mathrm{cm} \text { ) }\end{array}$} & \multirow{2}{*}{$\begin{array}{l}\text { Sub-bottom } \\
\text { depth } \\
\text { (m) }\end{array}$} & \multicolumn{4}{|c|}{ Bulk sediment } & \multicolumn{5}{|c|}{$<2 \mu \mathrm{m}$ fraction } \\
\hline & & Calcite & Siderite & $\begin{array}{c}\mathrm{Mg} \\
\text { calcite }\end{array}$ & Quartz & $\begin{array}{l}\text { Smec- } \\
\text { tite }\end{array}$ & Illite & $\begin{array}{l}\text { Kao- } \\
\text { linite }\end{array}$ & Chlorite & $\begin{array}{c}\text { Kaolinite }+ \\
\text { chlorite }\end{array}$ \\
\hline $2-1,43-46$ & 99.93 & & & & & 35 & 45 & 12 & 6 & \\
\hline $3-1,51-54$ & 109.51 & 64 & & & 4 & & & & & \\
\hline $3-2,51-54$ & 111.01 & & & & & 52 & 32 & 11 & 5 & \\
\hline $4-1,44-48$ & 118.94 & 61 & & & 5 & & & & & \\
\hline $4-2,44-48$ & 120.44 & & & & & 53 & 28 & 13 & 6 & \\
\hline $5-1,34-38$ & 128.34 & 76 & 2 & & 2 & & & & & \\
\hline $5-2,34-38$ & 129.84 & & & & & 25 & 49 & 13 & 13 & \\
\hline $6-1,44-48$ & 137.94 & 75 & & & 2 & & & & & \\
\hline $6-2,44-48$ & 139.44 & & & & & 37 & 45 & 12 & 6 & \\
\hline $7-1,50-55$ & 147.50 & 79 & & & 1 & & & & & \\
\hline $7-2,50-55$ & 149.00 & & & & & 21 & 51 & 14 & 14 & \\
\hline $8-1,72-77$ & 157.22 & 67 & & & 2 & & & & & \\
\hline $8-2,72-77$ & 158.12 & & & & & 32 & 40 & 14 & 14 & \\
\hline $9-1,80-85$ & 166.80 & 74 & & & 1 & & & & & \\
\hline $9-2,80-85$ & 167.58 & & & & & 38 & 38 & 8 & 16 & \\
\hline $10-1,31-33$ & 176.81 & 74 & & & 3 & & & & & \\
\hline $10-2,31-33$ & 177.31 & & & & & 16 & 54 & 15 & 15 & \\
\hline $11-1,26-28$ & 185.26 & 72 & & & 3 & & & & & \\
\hline $11-2,26-28$ & 186.76 & & & & & 18 & 48 & 17 & 17 & \\
\hline $12-1,20-22$ & 194.70 & 68 & & & 3 & & & & & \\
\hline $12-2,20-22$ & 196.20 & & & & & 38 & 41 & 7 & 14 & \\
\hline $13-1,12-16$ & 204.12 & & & & & 45 & 37 & 6 & 12 & \\
\hline $14-1,57-61$ & 214.07 & 76 & 2 & & 1 & & & & & \\
\hline $14-2,57-61$ & 215.57 & & & & & 57 & 28 & 9 & 6 & \\
\hline $15-1,35-39$ & 223.35 & & & & & 36 & 42 & 10 & 12 & \\
\hline $16-1,34-39$ & 233.84 & 15 & & & 1 & & & & & \\
\hline $16-2,34-38$ & 234.34 & & & & & 52 & 27 & 11 & 10 & \\
\hline $17-1,22-26$ & 242.22 & 77 & & & & & & & & \\
\hline $17-2,22-26$ & 243.72 & & & & & 54 & 29 & 10 & 7 & \\
\hline $18-1,25-29$ & 251.75 & 56 & & & 2 & & & & & \\
\hline $18-2,25-29$ & 253.25 & & & & & 54 & 30 & 9 & 7 & \\
\hline $19-1,35-39$ & 261.35 & 51 & & & 5 & & & & & \\
\hline $19-2,35-39$ & 263.30 & & & & & 60 & 26 & 9 & 5 & \\
\hline $21-1,52-59$ & 280.52 & 79 & & & 1 & & & & & \\
\hline $21-2,80-85$ & 282.30 & & & & & 61 & 24 & 10 & 5 & \\
\hline $22-1,22-24$ & 289.72 & 50 & & & 11 & & & & & \\
\hline $22-2,71-72$ & 291.71 & & & & & 67 & 25 & 12 & 6 & \\
\hline $23-1,11-13$ & 299.11 & 58 & & & 3 & & & & & \\
\hline $23-2,68-70$ & 301.18 & & & & & 78 & 14 & 4 & 4 & \\
\hline $25-1,33-35$ & 318.33 & 47 & & & 3 & & & & & \\
\hline $25-2,60-62$ & 320.10 & & & & & 81 & 11 & & & 8 \\
\hline $25-3,50-53$ & 321.50 & & & & & 74 & 13 & & & 13 \\
\hline $26-1,40-42$ & 327.90 & & & & & 73 & 15 & & & 12 \\
\hline $27-1,39-42$ & 337.39 & 36 & & & 6 & & & & & \\
\hline $27-2,39-42$ & 338.89 & & & & & 83 & 10 & & & 7 \\
\hline $28-1,47-50$ & 346.97 & 48 & & & 5 & & & & & \\
\hline $28-2,47-50$ & 348.47 & & & & & 76 & 13 & & & 11 \\
\hline $29-1,48-51$ & 356.48 & 38 & & & 6 & & & & & \\
\hline $29-2,48-51$ & 357.98 & & & & & 83 & 9 & & & 8 \\
\hline $30-1,47-49$ & 365.97 & 34 & & & 8 & & & & & \\
\hline $30-2,47-49$ & 367.47 & & & & & 80 & 13 & & & 7 \\
\hline $31-1,51-53$ & 375.51 & 44 & & & 7 & & & & & \\
\hline $31-2,51-53$ & 377.01 & & & & & 87 & 8 & & & 5 \\
\hline $32-1,69-71$ & 385.19 & 41 & & & 9 & & & & & \\
\hline $32-2,69-71$ & 386.69 & & & & & 91 & 7 & & & 2 \\
\hline $33-1,58-59$ & 394.58 & 40 & & & 6 & & & & & \\
\hline $33-2,58-59$ & 396.08 & & & & & 87 & 9 & & & 4 \\
\hline $34-1,61-62$ & 404.11 & & & & & 87 & 8 & & & 5 \\
\hline $34-2,61-62$ & 405.61 & 25 & & 10 & 6 & & & & & \\
\hline $34-4,61-62$ & 408.61 & 41 & & & 5 & & & & & \\
\hline $34-5,61-62$ & 410.11 & & & & & 79 & 16 & & & 5 \\
\hline $35-1,61-62$ & 413.61 & 13 & & & 5 & & & & & \\
\hline $35-2,61-62$ & 415.11 & & & & & 94 & 6 & & & \\
\hline
\end{tabular}

Note: Bulk sediment contained no dolomite, aragonite, or anhydrite. 


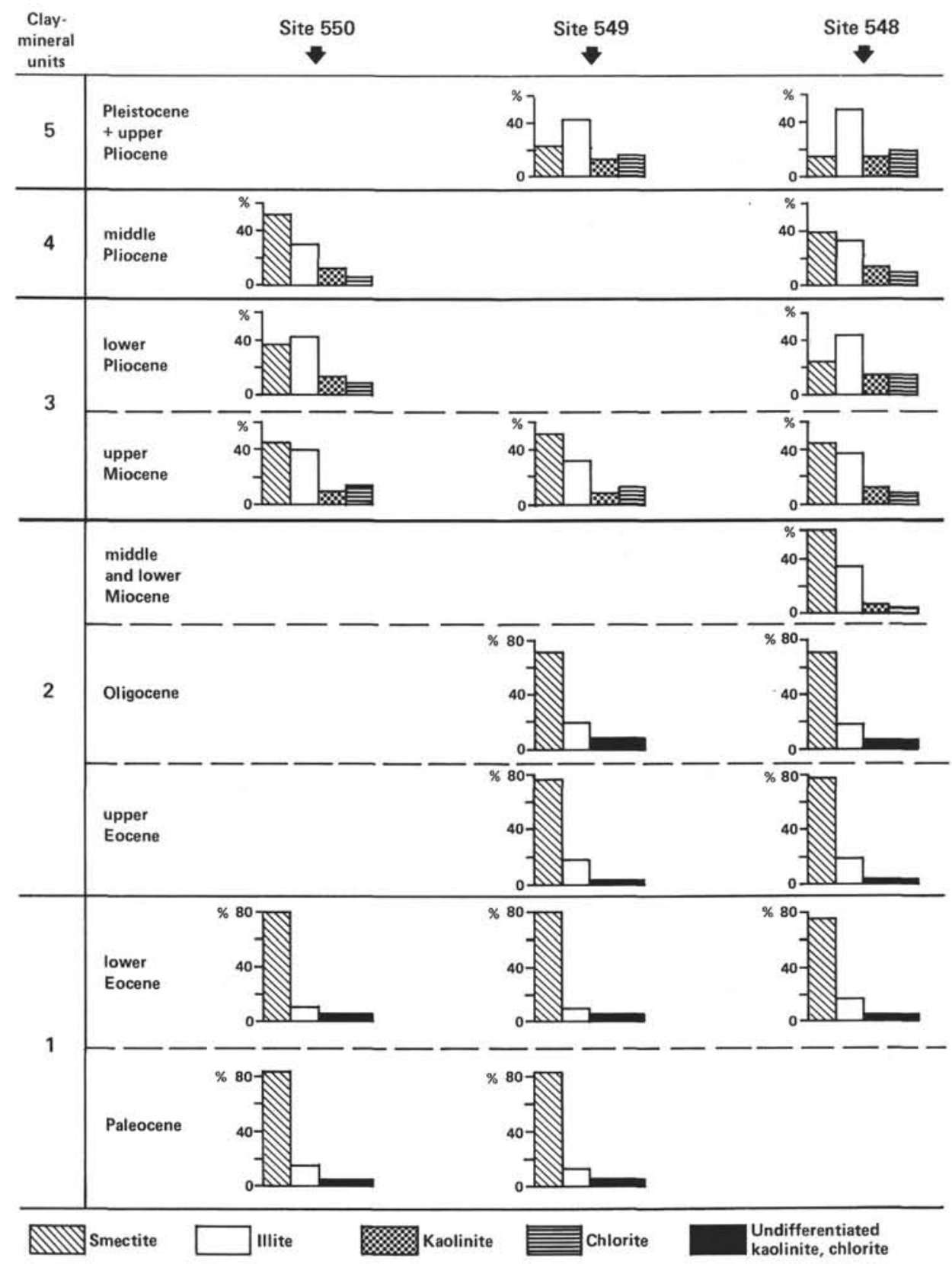

Figure 5. Diagram representing Cenozoic clay assemblages.

between Samples 6-2, 90-91 cm and 5-2, 70-71 cm; in Hole 550 between Samples 16-2, 34-38 cm and 15-1, $35-39 \mathrm{~cm}$ ).

2. A decrease in carbonate content and a massive increase in detrital-quartz content in Hole 548A (between Samples 12-1, 65-66 cm and 10-2, 72-73 cm).

These changes could be the result of several causes. First, they could be due to hydrodynamic events. In Hole $548 \mathrm{~A}$, the change corresponds to a stratigraphic gap recognized at the middle/upper Miocene contact (see site chapter). In Hole 549, the Neogene section is very thin and the stratigraphic series very incomplete. Numerous previous studies have already emphasized the frequency of hiatuses in the North Atlantic sedimentation (van Andel, 1975). A prominent gap in the lower middle Miocene has been especially attributed by Roberts and Mon- tadert (1979b) to "the erosion or non-deposition associated with the vigorous bottom circulation that developed the exchange of water between the Norwegian Sea and the North Atlantic Ocean." According to these authors, full exchange of waters across the Iceland Faeroes Ridge would have begun in post-middle Miocene time after the subsidence of the Ridge, namely, long after the opening of the Norwegian Sea in the early Eocene (Talwani and Eldholm, 1977). The observed increase of primary clay minerals-as typical minerals originating from colder climatic zones (Biscaye, 1965)-is consistent with the opinion of Roberts and Montadert. Moreover, the date of this increase closely corresponds to the epoch of the phenomena described by these authors.

A second possible cause would be a change of climate. The increase of primary clay minerals took place 
at the same time as the development of the East Antarctic ice cap and that of the South Atlantic cooling (Shackleton and Kennett, 1975). A temperature drop of the oceanic waters in the Northern Hemisphere is also reported in Leg 48 studies (Roberts and Montadert, 1979a).

The high post-early Miocene detrital-sediment accumulation rate $(>20 \mathrm{~m} / \mathrm{m}$.y.) calculated for Site 548 , the closest to the continent, could also indicate a climatic change, particularly a humidification, which could have entailed, from the middle Miocene, an increase of terrigenous input.

This terrigenous contribution becomes more abundant and coarser in the sediment record, whereas clay minerals become richer in illite and chlorite. Alterations on the neighboring lands favored less the smectite genesis, and this could signify a change to more humid and cooler climatic conditions. Only the finest fractions reached the deep-sea domain (Sites 550 and 549), and the coarse fractions were trapped on the margin (Site 548). Arguments concerning the climatic crisis hypothesis have been recently put forward in a comparative study of continental and deep-sea marine Neogene deposits in the northeastern Atlantic (Latouche and Maillet, 1982); it has been suggested that cooling and humidification of climate, specifically in the Britannic domain, took place during the Miocene. Data obtained from Leg 80 mineralogical studies agree well with this interpretation, and permit us to locate with more accuracy the major change at the middle/upper Miocene boundary.

In the Pliocene, encountered only at Sites 548 and 550 , the mineralogical composition of deposits becomes more variable, but indicates nevertheless another important change. Indeed, after a slight increase in smectite content (in Hole 548 between Samples 20-3, 33-36 cm and 17-4, 53-56 cm; in Hole 550 between Samples 4-2, $44-48 \mathrm{~cm}$ and $3-2,51-54 \mathrm{~cm}$ ), apparently associated with the reworking of underlying formations, the clay fraction becomes emphatically dominated by illite and chlorite in upper Pliocene and Pleistocene deposits. The relative abundances of the different minerals undergoupsection to the present-day deposits-numerous variations, which must evidently be related to the climatic fluctuations (Vergnaud Grazzini et al., 1979) reported for the end of the Cenozoic, after the development of the Arctic ice cap (Blank and Margolis, 1975). According to a pattern already established in the northeastern Atlantic (Alvinerie et al., 1978; Chamley, 1979), the strata richest in illite and chlorite should correspond to deposits laid down in a cold period, and those layers richer in smectite should correspond to warm periods of sedimentation.

\section{Comparison with Mineralogical Data from Legs $47 B$ and 48}

The general trend of the Cenozoic sedimentation in the northeastern Atlantic proposed here is in accordance with data from previous studies. Several stages that have been defined by mineralogical data are more or less contemporaneous with major geological events on a world- wide scale: Cretaceous/Tertiary or Eocene/Oligocene limits (Cavelier et al., 1981), Paleogene/Neogene boundary crisis (Latouche and Maillet, 1978, 1979), late Pliocene sharp temperature decrease (Vergnaud Grazzini et al., 1979). Nevertheless, the exact stratigraphic positions of some of these breaks-and particularly their significance-remain to be determined, for two important episodes recorded in the stratigraphic column have hitherto been inadequately investigated in northeastern Atlantic.

The first episode is the Oligocene, the sediment record of which is often absent or barely evident at previous DSDP sites. The second episode is the Quaternary, the record of which was very often poorly sampled because of technical problems. With the help of a comparatively continuous sampling of the Cenozoic series, Leg 80 allowed us to specify precisely mineralogical discontinuities already recognized from studies on Legs 47B and 48. Further, the Leg 80 results, spanning the entire northeastern Atlantic European margin, allow us to suggest a general pattern of the environmental evolution of this province during the Cenozoic. Hence Figure 6 , which shows the average distribution in time and space of components of typically primary terrigenous origin: quartz, illite, and chlorite. The three zones considered cover a north-south area: Rockall (Leg 48), Goban Spur (Leg 80), and west of Portugal (Leg 47B). The evolution of terrigenous materials appears to be governed by three

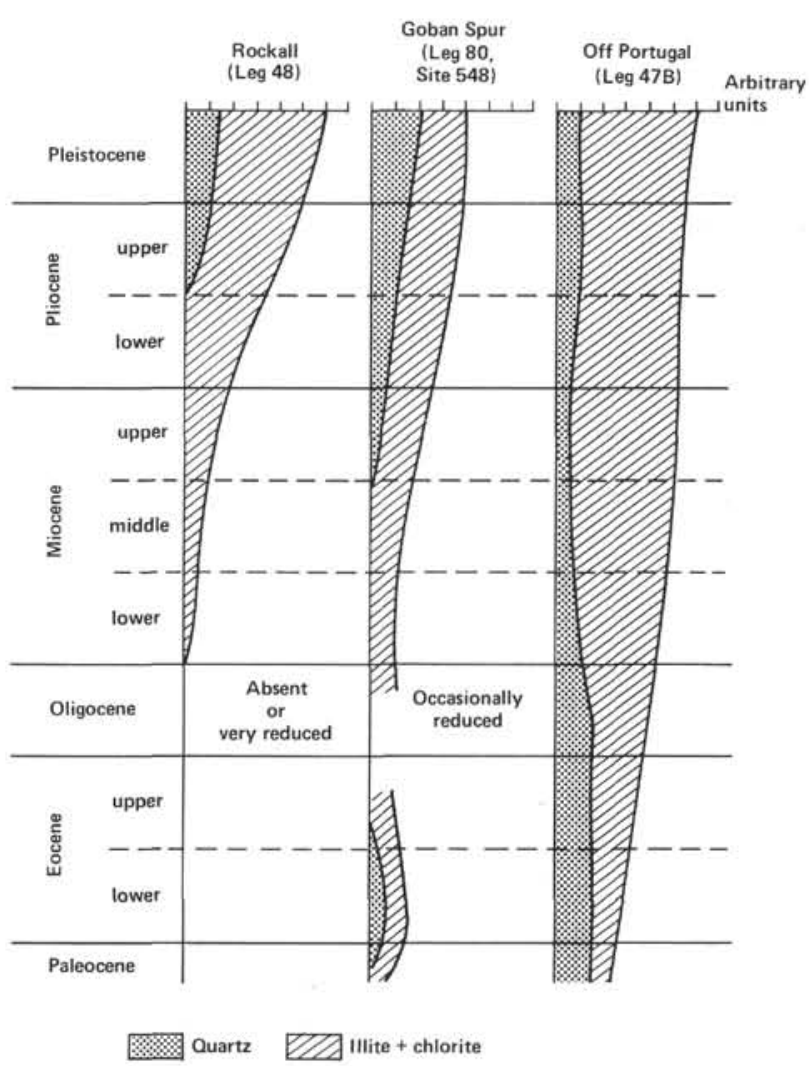

Figure 6. General evolution pattern of primary terrigenous minerals (quartz and illite + chlorite). 
factors: (1) continental factor: petrographic nature, morphology, and tectonic evolution; (2) climatic factor; and (3) hydrological factor.

In the Paleogene and through the top of the middle Miocene, terrigenous minerals of a quartz, illite, and chlorite type are present only in the southernmost zones (especially at Leg 47 sites).

The Rockall area is completely free of these minerals. Only continental areas bearing acid magmatic rocks (Pyrenees, Brittany, Ireland) dispose of these "primary" minerals; inputs were accentuated in the south by active Pyrenean tectonics, which gave rise to erosion, the products of which were for the main part trapped on the continent or nearshore environments (Latouche and Maillet, 1980) but which gave nevertheless an outflow of terrigenous materials to the sea. In fact, a postcruise survey in the Goban Spur Area revealed an intraplate compressional phase coeval with Pyrenean orogeny (Sibuet et al., this vol.) and with an uplifting of the Irish landmass (de Graciansky and Poag, this vol.). In the north (Rockall), contrarily, basic volcanic rocks could supply any of these primary materials. Furthermore, during the Paleocene and until the middle Miocene no exchange or hydrodynamic communication appears to have existed between these two petrographic domains: marine sedimentation was affected only by the neighboring continent.

Toward the top of the middle Miocene, illites and chlorites appear in the Rockall area and outnumber the smectites at Goban Spur and the Leg 47B area west of Portugal. A massive arrival of quartz is recorded on Goban Spur at the most coastal site (548). This quartz arrival indicates humidification of climate-a phenomenon that has not been observed in the other zones such as the north (Rockall), because of the petrographic nature of the neighboring areas (which cannot supply any quartz), or in the south, because of the history of tectonics in the zone, which was subjected to a "tectonic calm" after the Pyrenean paroxysmal phase of the Paleogene. The result of that calm was a relative diminishing of the supply of coarse materials in the south. It is also possible, as regards Rockall in the north, that the climatic evolution was less felt in the north than in the south (Latouche and Maillet, 1980), and has consequently favored erosion less.

The replacement of smectites by illites and chlorites appears to be closely associated with the climatic evolution of Goban Spur and western Portugal, both areas neighboring the emerged lands on which acid rocks constitute the sources of illites and chlorites under relatively moderate climatic conditions. In the Rockall area, by contrast, these primary mineral sources are nonexistent; thus, in this area, the allochthonous illites and chlorites could serve as evidence for changes in ocean hydrological structures and mineral inputs carried by marine currents-which should have attained their full intensity during the late middle Miocene (Roberts and Montadert, 1979b). On this assumption, the phenomenon we have described (presence of illites and chlorites) must be considered within the general framework of the evolu- tion of communications between the Norwegian Sea and the Atlantic and in light of the probable relationship between climatic and hydrological changes reported for the middle/upper Miocene boundary.

The massive and sudden arrival of quartz in the Rockall area toward the end of the Pliocene involves once again the allochthonous nature of minerals, but unlike the previous period, it concerns also the coarser components. Consequently, ice-rafting appears to have been the most likely transport agent of these materials. In the other zones, no such spectacular increase of these coarser mineral components has been noted, either because of a local terrigenous sedimentation yielding large quantities of quartz (within which eventual allochthonous inputs cannot be detected), or because of a regional location (namely western Portugal) outside the iceberg transport path.

\section{GEOCHEMICAL STUDY: SITES 548, 549, AND 550}

Few samples were available, and those available were often very rich in carbonate; separation and analysis of the clay fractions were therefore impossible. Geochemical study has been restricted to the analysis of the $\mathrm{HCl}-$ soluble phase. Approximately one sample per core has been analyzed.

\section{METHODS}

Samples were crushed and subjected to $1 \mathrm{~N} \mathrm{HCl}$. The solutions were poured into a gauged flask and the amounts of $\mathrm{Al}, \mathrm{Fe}, \mathrm{Mn}, \mathrm{Ca}$, $\mathrm{Mg}, \mathrm{Sr}, \mathrm{Na}$, and $\mathrm{K}$ were determined by atomic absorption spectrometry. Analytical data were treated by statistical multivariate analysis (by factor analysis in $R$ mode).

\section{Analytical Data}

Analytical data are set out in Table 4 (A-C). Variations of amounts are shown in Figure 7 (A-C). Ca, Sr, and carbonate contents vary in a similar manner. $\mathrm{Al}, \mathrm{Fe}$, $\mathrm{Mg}$, and $\mathrm{K}$ also have parallel variations: the higher amounts are in Paleocene, lower Eocene, and Pleistocene sediments - that is, those with the lowest carbonate contents. But a systematic opposite variation of these two families of elements does not occur: the higher amounts of $\mathrm{Al}, \mathrm{Fe}, \mathrm{Mg}, \mathrm{K}$ do not necessarily indicate the samples of lowest carbonate content.

Mn content shows both stratigraphic and topographic variations. Amounts decrease from the Paleocene to the Pliocene. The highest values occur in the continental rise Hole 550; the lowest values were recorded in the continental shelf Hole 548 .

\section{Statistical Multivariate Analysis: Factor Analysis}

Factor analysis is the most generally useful of the multivariate methods to describe complex relations among many variables in terms of simpler relations among fewer variables (Harman, 1960). The $R$-mode analysis examines the relationships between variables, and it provides new uncorrelated variables, called factor scores, that may be used for classification purposes. The Varimax solution (Kaiser, 1958) has been chosen here. 
Table 4. Analytical data. A. Site 548 (Holes 548 and 548A).

\begin{tabular}{|c|c|c|c|c|c|c|c|c|c|c|}
\hline $\begin{array}{c}\text { Sample } \\
\text { (interval in } \mathrm{cm} \text { ) }\end{array}$ & $\begin{array}{c}\text { Quartz } \\
(\%)\end{array}$ & $\begin{array}{c}\text { Total } \\
\text { carbonate } \\
(\%)\end{array}$ & $\begin{array}{c}\mathrm{Fe}_{2} \mathrm{O}_{3} \\
(\%)\end{array}$ & $\underset{(\%)}{\mathrm{Al}_{2} \mathrm{O}_{3}}$ & $\begin{array}{l}\mathrm{CaO} \\
(\%)\end{array}$ & $\begin{array}{l}\mathrm{MgO} \\
(\%)\end{array}$ & $\begin{array}{c}\mathrm{Na}_{2} \mathrm{O} \\
(\%)\end{array}$ & $\begin{array}{l}\mathrm{K}_{2} \mathrm{O} \\
(\%)\end{array}$ & $\begin{array}{c}\mathrm{Mn} \\
(\mathrm{ppm})\end{array}$ & $\underset{(\mathrm{ppm})}{\mathrm{Sr}}$ \\
\hline \multicolumn{11}{|l|}{ Hole 548} \\
\hline $1-3,25-26$ & 18 & 9 & 3.40 & 2.50 & 8.20 & 2.00 & 1.10 & 0.64 & 480 & 60 \\
\hline $2-3,41-42$ & 28 & 16 & 3.30 & 1.80 & 11.70 & 1.70 & 0.74 & 0.36 & 480 & 150 \\
\hline $2-6,53-54$ & 27 & 17 & 3.10 & 1.80 & 11.70 & 1.80 & 0.84 & 0.41 & 390 & 140 \\
\hline $3-2,56-57$ & 29 & 16 & 2.80 & 1.60 & 11.60 & 1.70 & 0.64 & 0.43 & 320 & 140 \\
\hline $4-3,75-76$ & 28 & 15 & 3.00 & 1.80 & 10.90 & 1.70 & 0.95 & 0.42 & 350 & 110 \\
\hline $5-3,49-50$ & 26 & 11 & 3.40 & 2.40 & 9.30 & 1.60 & 0.88 & 0.57 & 350 & 90 \\
\hline $6-3,28-29$ & 27 & 24 & 2.10 & 1.30 & 15.60 & 1.20 & 0.77 & 0.36 & 320 & 300 \\
\hline $7-4,84-85$ & 26 & 23 & 2.80 & 1.50 & 16.80 & 1.40 & 0.71 & 0.34 & 610 & 275 \\
\hline $8-3,90-91$ & 30 & 55 & 1.10 & 0.72 & 26.60 & 0.91 & 0.91 & 0.17 & 870 & 650 \\
\hline $9-2,47-48$ & 26 & 41 & 1.00 & 0.62 & 26.60 & 0.63 & 0.81 & 0.17 & 740 & 550 \\
\hline $10-3,43-44$ & 40 & 25 & 1.50 & 0.64 & 20.30 & 0.71 & 0.51 & 0.20 & 390 & 360 \\
\hline $11-4,73-74$ & 23 & 7 & 2.70 & 2.40 & 6.10 & 1.40 & 0.71 & 0.49 & 480 & 75 \\
\hline $13-2,130-131$ & 14 & 52 & 1.70 & 1.20 & 31.20 & 0.80 & 1.20 & 0.29 & 1100 & 530 \\
\hline $15-4,19-20$ & 26 & 32 & 1.70 & 0.87 & 24.90 & 0.83 & 0.78 & 0.22 & 420 & 440 \\
\hline $16-2,10-11$ & 27 & 10 & 2.10 & 1.80 & 9.60 & 1.20 & 0.64 & 0.44 & 420 & 150 \\
\hline $18-2,45-48$ & 33 & 12 & 1.70 & 1.10 & 10.90 & 0.96 & 0.68 & 0.30 & 260 & 210 \\
\hline $19-2,73-75$ & 28 & 33 & 0.83 & 0.55 & 22.20 & 0.66 & 0.51 & 0.17 & 160 & 525 \\
\hline $20-2,33-36$ & 20 & 38 & 1.00 & 0.74 & 26.30 & 0.63 & 0.71 & 0.21 & 230 & 590 \\
\hline $21-1,120-123$ & 20 & 33 & 0.72 & 0.55 & 27.80 & 0.66 & 0.71 & 0.18 & 160 & 725 \\
\hline $22-1,80-83$ & 30 & 38 & 0.64 & 0.55 & 24.20 & 0.63 & 0.61 & 0.16 & 130 & 590 \\
\hline $23-1,104-105$ & 20 & 39 & 0.86 & 0.76 & 28.90 & 0.71 & 0.64 & 0.21 & 230 & 700 \\
\hline $24-1,114-117$ & 22 & 38 & 0.64 & 0.59 & 25.40 & 0.58 & 0.61 & 0.17 & 130 & 600 \\
\hline $25-1,47-50$ & 23 & 41 & 0.68 & 0.59 & 26.40 & 0.58 & 0.64 & 0.18 & 130 & 690 \\
\hline $26-1,68-71$ & 30 & 38 & 0.64 & 0.53 & 26.10 & 0.71 & 0.57 & 0.17 & 130 & 660 \\
\hline $27-1,10-13$ & 19 & 42 & 0.64 & 0.68 & 28.80 & 0.58 & 0.57 & 0.17 & 190 & 740 \\
\hline $28-1,15-18$ & 26 & 39 & 0.47 & 0.38 & 28.10 & 0.42 & 0.44 & 0.10 & 130 & 625 \\
\hline $29-1,80-81$ & 12 & 50 & 0.72 & 0.81 & 33.70 & 0.55 & 0.54 & 0.17 & 160 & 800 \\
\hline $30-1,29-30$ & 6 & 19 & 2.00 & 0.53 & 17.50 & 0.33 & 0.20 & 0.10 & 160 & 425 \\
\hline $31-1,80-81$ & 28 & 48 & 0.61 & 0.64 & 32.60 & 0.50 & 0.47 & 0.14 & 160 & 740 \\
\hline $32-1,80-81$ & 20 & 48 & 0.64 & 0.68 & 32.90 & 0.58 & 0.47 & 0.15 & 160 & 890 \\
\hline $33-1,80-81$ & 14 & 47 & 0.61 & 0.59 & 35.10 & 0.50 & 0.51 & 0.14 & 190 & 875 \\
\hline \multicolumn{11}{|l|}{ Hole 548A } \\
\hline $2-1,107-112$ & 12 & 53 & 0.54 & 0.53 & 37.50 & 0.46 & 0.51 & 0.12 & 190 & 1010 \\
\hline $3-1,80-82$ & 18 & 41 & 0.64 & 0.64 & 30.10 & 0.50 & 0.47 & 0.15 & 130 & 690 \\
\hline $4-2,101-102$ & 15 & 48 & 0.50 & 0.55 & 44.90 & 0.46 & 0.40 & 0.11 & 160 & 760 \\
\hline $5-1,34-36$ & 17 & 26 & 0.93 & 0.87 & 21.00 & 0.55 & 0.57 & 0.18 & 130 & 500 \\
\hline $6-2,147-149$ & 18 & 43 & 0.50 & 0.45 & 32.50 & 0.46 & 0.37 & 0.10 & 160 & 650 \\
\hline $7-1,121-123$ & 28 & 38 & 0.50 & 0.36 & 26.10 & 0.42 & 0.34 & 0.10 & 60 & 525 \\
\hline $8-1,65-60$ & 22 & 46 & 0.47 & 0.43 & 25.80 & 0.38 & 0.34 & 0.10 & 60 & 510 \\
\hline $9-1,78-79$ & 19 & 36 & 0.64 & 0.68 & 29.30 & 0.46 & 0.37 & 0.14 & 130 & 575 \\
\hline $10-2,72-73$ & 28 & 34 & 0.57 & 0.53 & 25.10 & 0.46 & 0.44 & 0.13 & 100 & 500 \\
\hline $11-1,47-48$ & 16 & 47 & 0.40 & 0.45 & 30.50 & 0.38 & 0.51 & 0.11 & 100 & 625 \\
\hline $12-1,65-66$ & 3 & 64 & 0.40 & 0.53 & 45.90 & 0.46 & 0.61 & 0.12 & 160 & 950 \\
\hline $13-1,71-72$ & 2 & 75 & 0.26 & 0.74 & 44.10 & 0.38 & 0.78 & 0.10 & 320 & 900 \\
\hline $14-1,20-22$ & 3 & 72 & 0.26 & 0.38 & 48.30 & 0.42 & 0.34 & 0.10 & 190 & 900 \\
\hline $15-1,32-33$ & 1 & 71 & 0.36 & 0.45 & 48.00 & 0.30 & 0.68 & 0.11 & 320 & 1100 \\
\hline $16-1,49-50$ & 1 & 74 & 0.47 & 0.40 & 50.80 & 0.42 & 0.74 & 0.12 & 420 & 960 \\
\hline $17-1,13-14$ & 3 & 66 & 1.00 & 0.72 & 43.00 & 0.58 & 0.64 & 0.21 & 710 & 840 \\
\hline $18-1,40-41$ & 3 & 65 & 0.78 & 0.64 & 45.90 & 0.46 & 0.71 & 0.21 & 230 & 660 \\
\hline $19-1,51-53$ & 3 & 65 & 0.54 & 0.49 & 43.00 & 0.55 & 0.64 & 0.16 & 1230 & 725 \\
\hline $20-1,84-85$ & 4 & 59 & 0.61 & 0.66 & 39.20 & 0.50 & 0.88 & 0.25 & 580 & 625 \\
\hline $21-1,71-73$ & 6 & 58 & 0.64 & 0.66 & 35.00 & 0.55 & 0.64 & 0.17 & 680 & 600 \\
\hline $22-1,90-91$ & 6 & 60 & 0.76 & 0.68 & 38.90 & 0.58 & 0.61 & 0.17 & 810 & 610 \\
\hline $23-1,59-60$ & 13 & 16 & 1.30 & 1.60 & 13.30 & 0.88 & 0.88 & 0.26 & 390 & 300 \\
\hline $24-1,41-42$ & 12 & 16 & 2.10 & 1.50 & 12.70 & 0.88 & 0.91 & 0.32 & 480 & 275 \\
\hline $25-1,31-32$ & 10 & 12 & 2.40 & 2.00 & 10.40 & 0.96 & 0.98 & 0.20 & 520 & 240 \\
\hline $27-1,59-60$ & 10 & 37 & 2.00 & 1.00 & 27.30 & 0.71 & 0.64 & 0.23 & 1160 & 475 \\
\hline $28-1,61-62$ & 15 & 26 & 1.60 & 1.20 & 19.00 & 0.80 & 0.68 & 0.10 & 1480 & 350 \\
\hline
\end{tabular}

\section{Varimax Matrix, $R$ Mode (Table 5)}

The eight variables may be reduced to four factors; the most important of these is the factor 1 .

Factor 1 uses $55 \%$ of total variance resulting from $\mathrm{Ca}-\mathrm{Sr}$ covariance, $\mathrm{Al}-\mathrm{Fe}-\mathrm{Mg}-\mathrm{K}$ covariance, and the statistically inverse variation of these two groups of variables. This lithologic factor is the consequence of the complementary variation of carbonate and argillaceous components.

Factor 4 exhibits $\mathrm{Ca}-\mathrm{Sr}$ residual variance not included in factor 1 . It particularizes the magnesian component of ferromagnesian calcite-bearing limestones, and also the "unclean" limestones releasing $\mathrm{Al}, \mathrm{Fe}, \mathrm{Mg}$, and $\mathrm{K}$ by $\mathrm{HCl}$ leaching, whereas the "clean" limestones are in factor 1 . This factor 4 expresses quantitatively that
$\mathrm{Ca}-\mathrm{Sr}$ variation is not systematically opposite to $\mathrm{Al}-\mathrm{Fe}-$ $\mathrm{Mg}-\mathrm{K}$ variation, as already observed. Mn does not occur in factor 1 , because the amounts are independent of the lithology.

Factor 2 shows a light $\mathrm{Mn}-\mathrm{Na}$ binding because of a light Mn-smectite correlation.

Factor 3 corresponds to the independent variation of $\mathrm{Na}$. Factor-scores logging is shown in Figure $8(\mathrm{~A}-\mathrm{C})$.

\section{Lithologic Factors 1 and 4}

Samples are plotted in the plane of lithologic factors 1 and 4 in Figure 9. Ante-Miocene sediments are grouped according to stratigraphic entities, since the amounts of carbonates, low in the Paleocene and lower Eocene, increase up to the Oligocene. The limestones appear "clean" except in four siderite-bearing samples. Paleo- 
Table 4. Analytical data. B. Site 549 (Holes 549 and 549A).

\begin{tabular}{|c|c|c|c|c|c|c|c|c|c|c|}
\hline $\begin{array}{c}\text { Sample } \\
\text { (interval in cm) }\end{array}$ & $\begin{array}{c}\text { Quartz } \\
(\%)\end{array}$ & $\begin{array}{c}\text { Total } \\
\text { carbonate } \\
(\%)\end{array}$ & $\begin{array}{c}\mathrm{Fe}_{2} \mathrm{O}_{3} \\
(\%)\end{array}$ & $\underset{(\%)}{\mathrm{Al}_{2} \mathrm{O}_{3}}$ & $\begin{array}{l}\mathrm{CaO} \\
(\%)\end{array}$ & $\begin{array}{c}\mathrm{MgO} \\
(\%)\end{array}$ & $\begin{array}{c}\mathrm{Na}_{2} \mathrm{O} \\
(\%)\end{array}$ & $\begin{array}{l}\mathrm{K}_{2} \mathrm{O} \\
(\%)\end{array}$ & $\begin{array}{c}\mathrm{Mn} \\
(\mathrm{ppm})\end{array}$ & $\begin{array}{c}\mathrm{Sr} \\
(\mathrm{ppm})\end{array}$ \\
\hline \multicolumn{11}{|l|}{ Hole $549 \mathrm{~A}$} \\
\hline $4-1,80-81$ & 4 & 70 & 1.00 & 1.10 & 44.50 & 0.63 & 0.68 & 0.23 & 260 & 1010 \\
\hline $5-1,70-71$ & 4 & 80 & 0.72 & 0.77 & 45.90 & 0.58 & 0.95 & 0.18 & 190 & 925 \\
\hline $6-1,90-91$ & 2 & 76 & 0.64 & 0.87 & 42.00 & 0.63 & 0.88 & 0.20 & 390 & 940 \\
\hline $7-1,60-61$ & 1 & 73 & 0.61 & 0.59 & 48.00 & 0.50 & 0.81 & 0.19 & 740 & 960 \\
\hline $8-1,70-71$ & 2 & 75 & 0.76 & 0.64 & 46.20 & 0.59 & 1.00 & 0.18 & 550 & 1000 \\
\hline $9-1,70-71$ & 1 & 79 & 0.40 & 0.36 & 48.70 & 0.38 & 0.95 & 0.11 & 480 & 1125 \\
\hline $10-1,60-61$ & 1 & 77 & 0.43 & 0.43 & 47.30 & 0.38 & 0.88 & 0.11 & 680 & 1125 \\
\hline $11-1,20-23$ & 2 & 79 & 0.43 & 0.38 & 47.60 & 0.46 & 1.00 & 0.10 & 645 & 1050 \\
\hline $12-1,20-23$ & 1 & 79 & 0.36 & 0.25 & 48.30 & 0.42 & 0.91 & 0.10 & 810 & 910 \\
\hline $13-1,20-23$ & 1 & 75 & 0.64 & 0.36 & 47.30 & 0.46 & 0.91 & 0.10 & 810 & 1060 \\
\hline $14-1,20-23$ & 1 & 80 & 0.36 & 0.34 & 47.30 & 0.33 & 0.96 & 0.10 & 680 & 1110 \\
\hline $15-1,20-23$ & 2 & 78 & 0.40 & 0.45 & 42.40 & 0.42 & 0.98 & 0.12 & 710 & 1010 \\
\hline $16-1,80-83$ & 1 & 78 & 0.29 & 0.28 & 47.30 & 0.33 & 0.81 & 0.10 & 710 & 1150 \\
\hline $17-1,20-23$ & 1 & 78 & 0.36 & 0.43 & 47.30 & 0.38 & 0.88 & 0.10 & 840 & 1090 \\
\hline $18-1,27-30$ & 1 & 78 & 0.19 & 0.25 & 48.70 & 0.38 & 0.81 & 0.10 & 740 & 1060 \\
\hline $20-1,40-41$ & 1 & 79 & 0.40 & 0.40 & 45.90 & 0.38 & 0.74 & 0.11 & 610 & 1025 \\
\hline $24-1,20-23$ & 1 & 81 & 0.29 & 0.28 & 49.40 & 0.38 & 0.78 & 0.10 & 680 & 1010 \\
\hline $25-1,20-23$ & 1 & 71 & 0.36 & 0.38 & 51.50 & 0.42 & 0.81 & 0.10 & 770 & 1025 \\
\hline $28-1,20-23$ & 1 & 69 & 0.47 & 0.53 & 50.10 & 0.46 & 0.81 & 0.11 & 840 & 950 \\
\hline $33-1,60-61$ & 1 & 72 & 0.43 & 0.38 & 46.20 & 0.42 & 0.64 & 0.10 & 1100 & 840 \\
\hline $34-1,80-81$ & 2 & 68 & 0.43 & 0.47 & 46.60 & 0.42 & 0.68 & 0.12 & 810 & 950 \\
\hline $36-1,5-7$ & 1 & 73 & 0.43 & 0.43 & 42.70 & 0.42 & 0.68 & 0.10 & 810 & 900 \\
\hline $38-1,34-37$ & 2 & 66 & 0.36 & 0.40 & 45.20 & 0.42 & 0.84 & 0.10 & 940 & 910 \\
\hline $40-1,40-41$ & 2 & 64 & 0.64 & 0.55 & 43.10 & 0.46 & 0.81 & 0.11 & 900 & 875 \\
\hline \multicolumn{11}{|l|}{ Hole 549} \\
\hline $2-1,24-27$ & 2 & 65 & 0.54 & 0.59 & 43.10 & 0.46 & 0.68 & 0.10 & 840 & 875 \\
\hline $4-1,72-73$ & 2 & 56 & 0.47 & 0.49 & 42.00 & 0.46 & 0.71 & 0.10 & 1030 & 850 \\
\hline $5-1,73-74$ & 3 & 84 & 0.47 & 0.45 & 41.00 & 0.46 & 0.57 & 0.10 & 1130 & 860 \\
\hline $6-1,66-67$ & 3 & 55 & 0.43 & 0.47 & 35.40 & 0.46 & 0.74 & 0.11 & 940 & 790 \\
\hline $7-1,30-32$ & 4 & 64 & 0.54 & 0.57 & 35.70 & 0.46 & 0.57 & 0.11 & 1030 & 750 \\
\hline $8-1,73-75$ & 4 & 69 & 0.57 & 0.55 & 37.50 & 0.46 & 0.57 & 0.12 & 770 & 650 \\
\hline $9-1,36-37$ & 4 & 70 & 0.47 & 0.49 & 36.40 & 0.42 & 0.61 & 0.14 & 1100 & 775 \\
\hline $10-1,44-46$ & 2 & 64 & 0.76 & 0.62 & 36.10 & 0.55 & 0.64 & 0.21 & 900 & 725 \\
\hline $11-1,101-104$ & 5 & 45 & 1.10 & 0.66 & 27.30 & 0.55 & 0.51 & 0.17 & 2190 & $\$ 25$ \\
\hline $12-1,47-49$ & 7 & 34 & 1.60 & 0.93 & 21.60 & 0.66 & 0.88 & 0.16 & 1390 & 400 \\
\hline $13-1,50-52$ & 3 & 57 & 0.83 & 0.57 & 32.80 & 0.46 & 0.64 & 0.13 & 2060 & 625 \\
\hline $14-1,51-53$ & 5 & 47 & 2.10 & 1.20 & 28.40 & 0.71 & 0.84 & 0.26 & 900 & 460 \\
\hline $15-1,51-53$ & 9 & 40 & 1.50 & 1.20 & 24.20 & 0.75 & 0.71 & 0.20 & 139 & 400 \\
\hline $16-1,51-52$ & 9 & 42 & 2.00 & 1.30 & 23.80 & 0.80 & 0.71 & 0.20 & 1550 & 440 \\
\hline $17-1,61-62$ & 3 & 47 & 1.70 & 1.20 & 25.80 & 0.88 & 0.91 & 0.17 & 1420 & 510 \\
\hline $18-1,60-61$ & 2 & 42 & 2.10 & 1.70 & 27.00 & 1.00 & 0.95 & 0.17 & 1260 & 500 \\
\hline $19-1,9-10$ & 2 & 41 & 1.60 & 1.20 & 24.90 & 0.80 & 1.10 & 0.20 & 1480 & 475 \\
\hline $20-1,65-67$ & 4 & S1 & 1.20 & 1.20 & 31.90 & 0.75 & 0.61 & 0.24 & 1840 & 590 \\
\hline
\end{tabular}

Table 4. Analytical data. C. Site 550 (Hole 550).

\begin{tabular}{|c|c|c|c|c|c|c|c|c|c|c|}
\hline $\begin{array}{c}\text { Sample } \\
\text { (interval in } \mathrm{cm} \text { ) }\end{array}$ & $\begin{array}{l}\text { Quartz } \\
(\%)\end{array}$ & $\begin{array}{c}\text { Total } \\
\text { carbonate } \\
(\%)\end{array}$ & $\begin{array}{c}\mathrm{Fe}_{2} \mathrm{O}_{3} \\
(\%)\end{array}$ & $\underset{(\%)}{\mathrm{Al}_{2} \mathrm{O}_{3}}$ & $\begin{array}{l}\mathrm{CaO} \\
(\%)\end{array}$ & $\begin{array}{c}\mathrm{MgO} \\
(\%)\end{array}$ & $\begin{array}{c}\mathrm{Na}_{2} \mathrm{O} \\
(\%)\end{array}$ & $\begin{array}{l}\mathrm{K}_{2} \mathrm{O} \\
(\%)\end{array}$ & $\begin{array}{c}\mathrm{Mn} \\
(\mathrm{ppm})\end{array}$ & $\begin{array}{c}\mathrm{Sr} \\
(\mathrm{ppm})\end{array}$ \\
\hline $3-1,51-54$ & 4 & 64 & 0.90 & 0.93 & 40.6 & 0.66 & 1.00 & 0.22 & 580 & 940 \\
\hline $4-1,44-48$ & 5 & 61 & 1.00 & 0.95 & 39.9 & 0.66 & 0.88 & 0.24 & 610 & 875 \\
\hline $5-1,34-38$ & 2 & 78 & 0.54 & 0.55 & 47.3 & 0.46 & 0.84 & 0.12 & 450 & 1040 \\
\hline $6-1,44-48$ & 2 & 75 & 0.43 & 0.47 & 48.0 & 0.46 & 0.81 & 0.11 & 350 & 1075 \\
\hline $7-1,50-55$ & 1 & 79 & 0.43 & 0.43 & 48.7 & 0.42 & 0.88 & 0.10 & 480 & 1160 \\
\hline $8-1,72-77$ & 2 & 67 & 0.43 & 0.47 & 46.6 & 0.42 & 0.81 & 0.12 & 390 & 1075 \\
\hline $9-1,80-85$ & 1 & 74 & 0.43 & 0.36 & 46.9 & 0.33 & 0.71 & 0.10 & 350 & 1075 \\
\hline $10-1,31-33$ & 3 & 74 & 0.54 & 0.47 & 46.6 & 0.38 & 0.78 & 0.11 & 580 & 1160 \\
\hline $11-1,26-28$ & 3 & 72 & 0.40 & 0.38 & 44.8 & 0.33 & 0.81 & 0.10 & 550 & 1100 \\
\hline $12-1,20-22$ & 3 & 68 & 0.50 & 0.53 & 43.8 & 0.38 & 0.78 & 0.12 & 480 & 1100 \\
\hline $14-1,57-61$ & 1 & 78 & 0.29 & 0.28 & 49.0 & 0.30 & 0.68 & 0.10 & 770 & 1160 \\
\hline $16-1,34-39$ & 1 & 78 & 0.36 & 0.38 & 49.4 & 0.30 & 0.74 & 0.10 & 1100 & 1200 \\
\hline $17-1,22-26$ & & 77 & 0.21 & 0.25 & 50.4 & 0.33 & 1.00 & 0.10 & 870 & 1310 \\
\hline $18-1,25-29$ & 2 & 66 & 0.43 & 0.53 & 42.4 & 0.46 & 0.71 & 0.14 & 1000 & 1100 \\
\hline $19-1,35-39$ & 3 & 51 & 0.72 & 0.81 & 34.0 & 0.66 & 0.81 & 0.23 & 810 & 875 \\
\hline $21-1,52-59$ & 1 & 79 & 0.21 & 0.30 & 48.7 & 0.30 & 0.68 & 0.10 & 1130 & 1290 \\
\hline $22-1,22-24$ & 11 & 50 & 1.70 & 1.80 & 4.9 & 1.00 & 1.20 & 0.43 & 390 & 150 \\
\hline $23-1,11-13$ & 3 & 88 & 0.57 & 0.66 & 37.5 & 0.63 & 0.78 & 0.15 & 2060 & 975 \\
\hline $25-1,33-35$ & 3 & 47 & 0.76 & 0.73 & 28.7 & 0.50 & 0.81 & 0.21 & 1350 & 525 \\
\hline $27-1,39-42$ & 6 & 36 & 0.83 & 2.50 & 24.2 & 0.66 & 0.91 & 0.19 & 1580 & 425 \\
\hline $28-1,47-50$ & 8 & 48 & 0.61 & 0.60 & 29.8 & 0.42 & 0.68 & 0.14 & 1680 & 610 \\
\hline $29-1,48-51$ & 6 & 38 & 0.54 & 0.57 & 23.5 & 0.38 & 0.88 & 0.21 & 1480 & 475 \\
\hline $30-1,47-49$ & 8 & 34 & 0.83 & 0.74 & 22.1 & 0.50 & 0.78 & 0.20 & 1520 & 490 \\
\hline $31-1,51-53$ & 7 & 44 & 2.70 & 1.20 & 26.3 & 0.71 & 0.71 & 0.23 & 2130 & 500 \\
\hline $32-1,69-71$ & & & 2.00 & 1.20 & 25.9 & 0.66 & 0.84 & 0.22 & 2930 & 500 \\
\hline $33-1,58-59$ & & & 2.30 & 1.20 & 27.0 & 0.71 & 0.81 & 0.25 & 2930 & 540 \\
\hline $34-2,61-62$ & & & 1.50 & 0.96 & 23.5 & 0.66 & 0.88 & 0.22 & 2770 & 525 \\
\hline $34-4,61-62$ & & & 0.97 & 0.90 & 25.6 & 0.63 & 0.91 & 0.25 & 3160 & 575 \\
\hline $35-1,61-62$ & & & 1.20 & 1.30 & 10.2 & 0.83 & 1.50 & 0.32 & 2610 & 225 \\
\hline
\end{tabular}




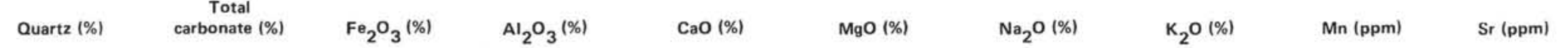

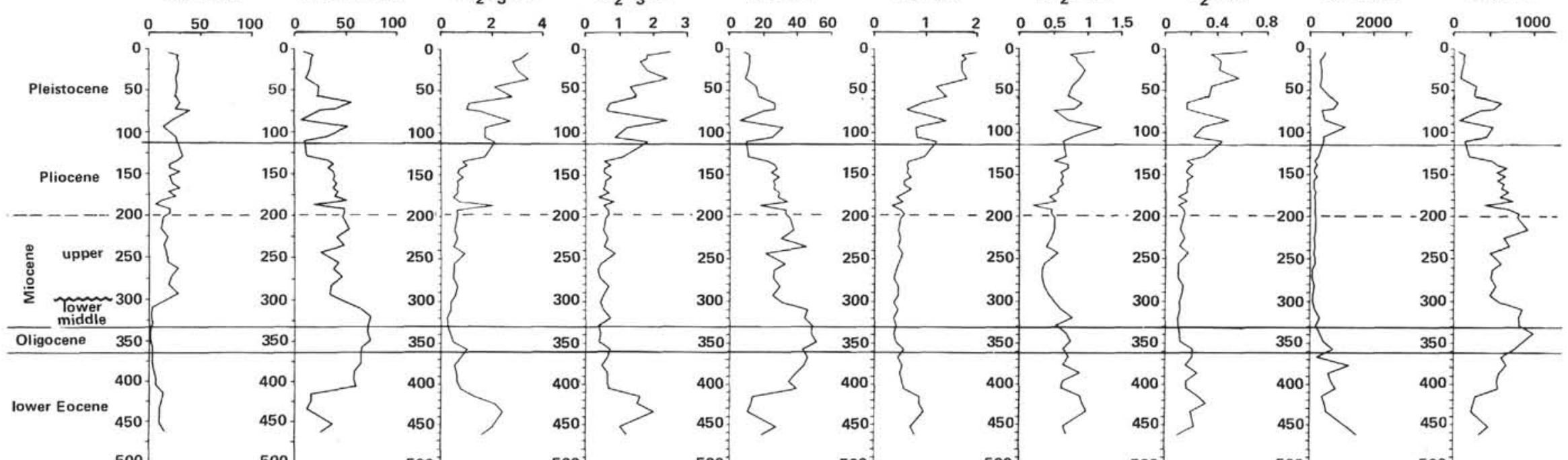

500

500

500

500

$500^{3}$

$500^{3}$

500

$500^{-}$

$500^{3}$

B

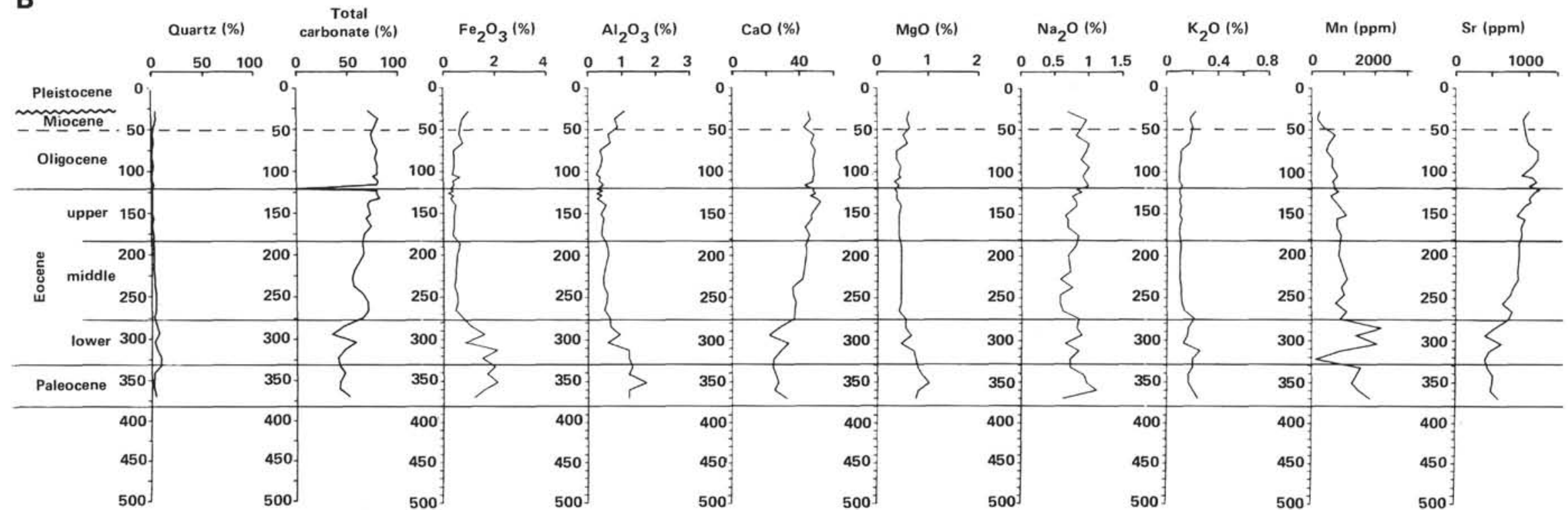




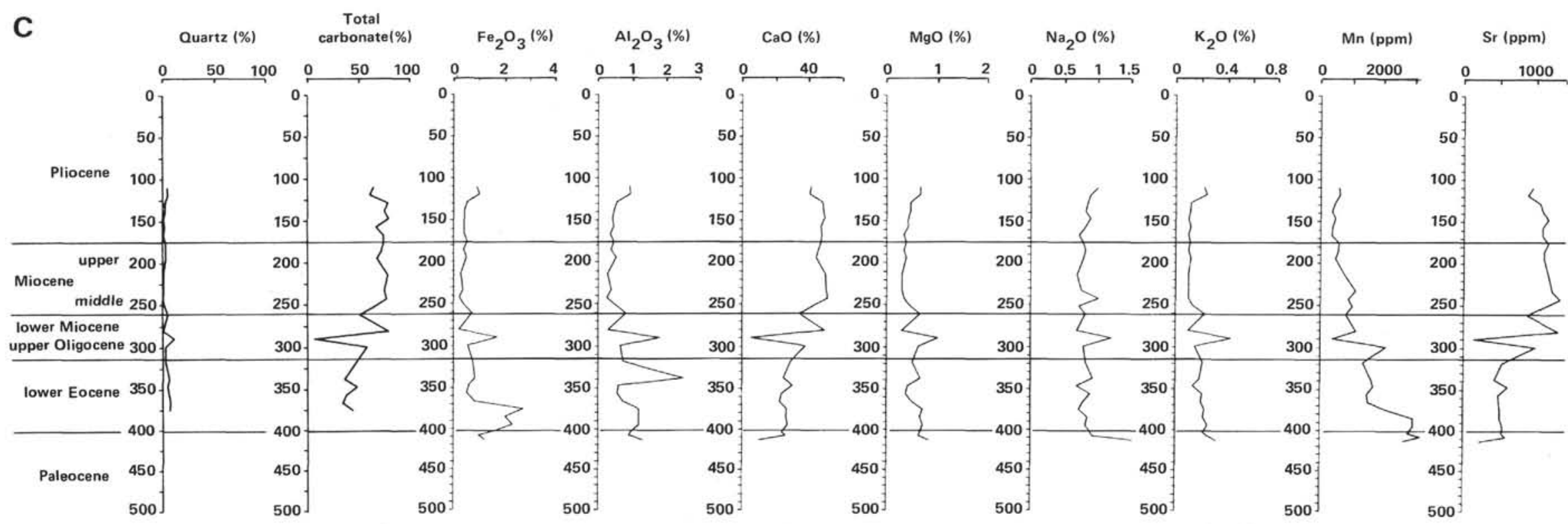

Figure 7. Analytical data. Left scales show sub-bottom depth (m). A. Site 548 (Holes 548 and 548A). B. Site 549 (Holes 549A and 549). C. Site 550 (Hole 550). 

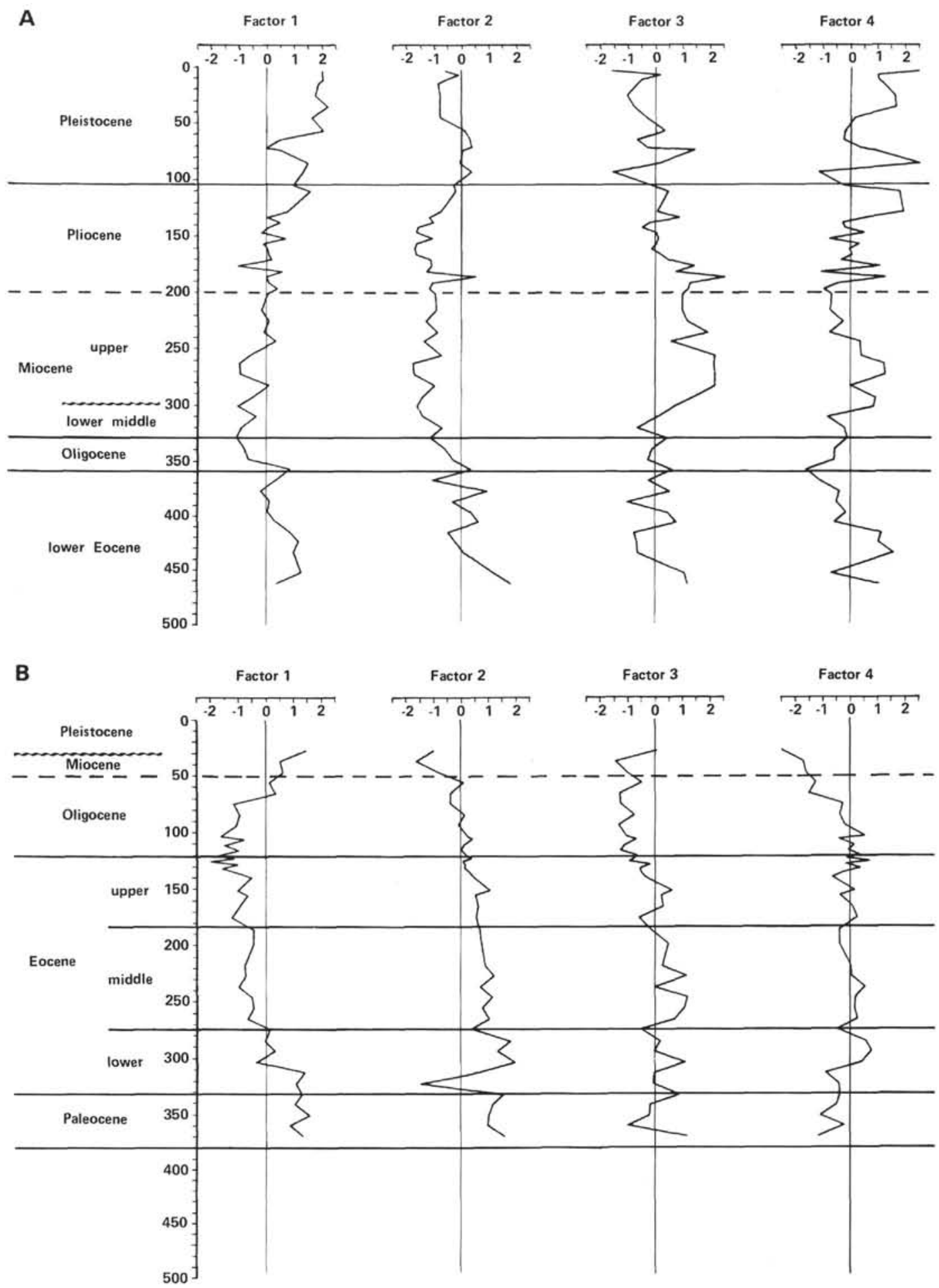

Figure 8. Factor analysis, factor-scores logging. Left scales show sub-bottom depth (m). A. Site 548 (Holes 548 and 548A). B. Site 549 (Holes 549A and 549). C. Site 550 (Hole 550).

cene and basal Eocene samples from Hole 550 are less "clean" than those from the lower Eocene; they are more argillaceous.

In Hole 550 the Miocene and Pliocene are not distinct from the Oligocene and the upper Eocene. This continuity of calcareous sedimentation is not recorded in Hole 548, where the sediments are more argillaceous and more illitic.

\section{Mn Factor}

The plot of samples in the plane of factors 1 and 2 shows that there is no relationship between the amounts 


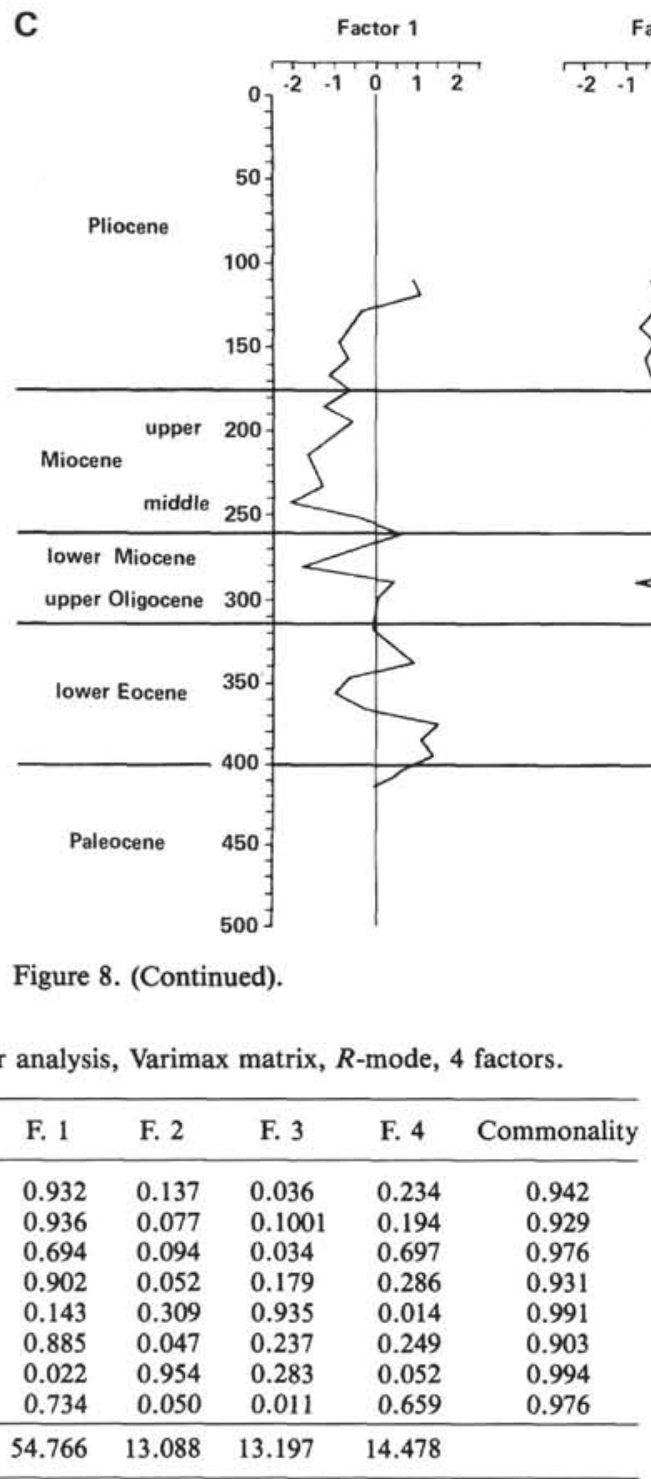

of Mn and the lithology (Fig. 10). The higher percentages of Mn occur in Paleocene and lower Eocene samples. From the middle Eocene upward, the distribution of points is controlled by the topographic situation: the samples from Hole 550 are more highly graded in $\mathrm{Mn}$ than those from Holes 548 and 549, especially in the Miocene and Pliocene sediments. Samples of upper Pliocene sediments have features intermediate between those of the Pliocene and the Pleistocene.

\section{Conclusions}

The conditions of sedimentation change at the top of the lower Eocene: below, the muds are argillaceous and highly manganesian, and from the top of the lower Eocene upward they become richer in carbonate and poorer in manganese. A second change occurs in the Miocene. This results from the bathymetric location: samples from Hole 550 are all rich in carbonate and manganese, whereas those from Hole 548 are more argillaceous, more illitic, and very weakly manganesian.
At Site 548, there is a third change in the upper Miocene, to a sediment with a larger clay fraction.

\section{PETROGRAPHIC ANALYSIS OF PEBBLES}

\section{SAMPLES STUDIED AND METHODOLOGY}

Occasional pebbles (13) were found within upper Cenozoic sediment levels at Sites 548 and 550, and within Mesozoic levels at Site 549. We interpret most as ice-rafted material (Sites 548 and 550), some as detrital pebbles (Site 549), and one as a diagenetic nodule (Site 548).

A standard petrographic thin section was cut from each sample, and petrographic determination was made using an optical polarizing microscope. For three of the pebbles, photomicrographs are included (Plates 1-3).

\section{Results}

\section{Site 548}

In Hole 548 , nine ice-rafted pebbles and one diagenetic in situ nodule were found within Units 1, 2, and 3 (see Fig. 2):

Sample 1-2, 65-66 cm (2.15-2.16 m sub-bottom). This pebble has a rounded shape with dimensions $1.5 \times 1 \times$ $1 \mathrm{~cm}$; it is dark brownish gray. In thin section we determined a biotite hornfels with a very fine-grained granoblastic structure. It contains $85 \%$ quartz and $15 \%$ interstitial biotite plus accessory minerals such as epidote, green hornblende, and pyrite; sedimentary structures (laminations) are still visible, underlined by biotite.

Sample 2-5, 70-71 cm (10.70-10.71 m sub-bottom) (Plate 1). This pebble has a rounded, elongated shape, with dimensions $2 \times 1 \times 1 \mathrm{~cm}$; it is dark grayish green. In thin section we determined a calk-alkaline syenite with a medium-grained granoblastic structure. It is very altered. 
It contains $65 \%$ feldspars, orthoclase, and unidentified plagioclase, both very altered to sericite, $15 \%$ uralitized pyroxene, $10 \%$ green hornblende, and $5 \%$ interstitial quartz; accessory minerals are small apatite crystals, small patches or veinlets of epidote, and titanomagnetite.

Sample 5-3, 85-87 cm (36.35-36.37 m sub-bottom). This pebble has a rounded shape with dimensions $1.5 \times$ $1 \times 1 \mathrm{~cm}$; it is medium grayish brown. In thin section we determined a fine-grained sandstone with abundant clay matrix; it is compact, slightly micaceous, and contains abundant small dark organic debris; some siderite occurs as diffuse cement, and pyrite occurs in small patches.

Sample 7-1, 91-92 cm (52.41-52.42 m sub-bottom). This pebble has a subrounded shape with dimensions $0.5 \times 0.5 \times 0.5 \mathrm{~cm}$; it is dark brown. In thin section we determined a fine-grained pyrite nodule with small veinlets or interstitial vugs filled with microcrystalline silica.

Sample 9-1, 43-44 cm (70.93-70.94 m sub-bottom). This pebble has a subangular to subrounded shape with dimensions $1 \times 1 \times 1 \mathrm{~cm}$; it is light brownish gray. In thin section we determined a medium-grained, well-sorted sandstone; it is cemented almost entirely by authigenic silica; only small amounts of detrital clays and micas are observed between the quartz grains.

Sample 10-1, 60-65 cm (71.10-71.15 m sub-bottom). This pebble has a rounded shape with dimensions $3 \times 2$ $\times 1.5 \mathrm{~cm}$; it is dark greenish gray. In thin section we determined a micaschist with biotite, muscovite, and garnet; it is very fresh and not altered. It exhibits a finegrained lepidoblastic structure, and is made up of quartz $(70 \%)$, plagioclase $(16 \%)$, biotite $(10 \%)$, muscovite $(3 \%)$, and large porphyroblasts of garnet $(1 \%)$.

Sample 10-1, 60-65 cm (71.10-71.15 m sub-bottom). This second pebble from the previous sample has a rounded shape with dimensions $3 \times 2 \times 1.5 \mathrm{~cm}$; it is dark grayish black. In thin section we determined a very finegrained, slightly shaly sandstone with silica and carbonate cement; it contains some small dark coaly and micaceous clay laminations and some gypsum flecks.

Sample 11-3, 120-121 cm (84.20-84.21 m sub-bottom) (Plate 2). This pebble has a rounded shape with dimensions $2 \times 1.5 \times 1.5 \mathrm{~cm}$; it is light brownish white. In thin section we determined a calcareous biomicrite containing some small detrital quartz grains and having small fractures and intergranular vugs filled with silty clay; some grains of glauconite are also present. The microfauna consists of blue encrusting derms (c), red algae (abt), gastropods (ex), echinoderms (c), bryozoans (abt), rotaliids and rupertiids (c), marginulinids (ex), quinqueloculinids (r), ophthalmidiids (ex), corals (ex), and lamellar pelecypods (ex). Code: ex $=1, r=3-4$, $c=5-9$, abt $=10$ specimens.

The pebble is Tertiary (occurrence of rupertiids and yellowish aspect of some tests), probably Miocene to Recent. Depositional environment: shallow peri-reef conditions in a photic (10-40 m) and low-energy zone close to a high-energy zone. Distal terrigenous influx inconvenient for corals, better for bryozoans. Normal oxygenation and seafloor circulation.
Sample 15-4, $110 \mathrm{~cm}$ (105.60 m sub-bottom). This pebble has a subangular to subrounded shape with dimensions $2 \times 1.5 \times 1.5 \mathrm{~cm}$; it is dark greenish gray. In thin section we determined a compact silicified silty shale with abundant small detrital micas, some heavy minerals (amphibole, sphene, zircon), abundant black organic fragments, and pyrite grains.

Sample 20-2, 6-7 cm (136.06-136.07 m sub-bottom). This pebble has a subrounded shape with dimensions $1.5 \times 1 \times 1 \mathrm{~cm}$; it is dark grayish black. In thin section we determined a microcrystalline siliceous and pyritic nodule with numerous ghosts of silicified organisms (foraminifers or radiolarians). It can be interpreted as an in situ diagenetic nodule associated with local organic activity and reducing conditions.

\section{Site 549}

In Hole 549, two pebbles were found within Unit 10 and more precisely, Subunit $10 \mathrm{~b}$. Though belonging to Mesozoic sediment levels, they have been included in this study because, in fact, they are the sole pebbles recovered in this hole. They have a terrigenous detrital origin. They are as follows:

Sample 78-1, 106-107 cm (844.06-844.07 m sub-bottom). This pebble has a subrounded to subangular shape with dimensions $2.5 \times 2 \times 1.5 \mathrm{~cm}$; it is medium grayish brown. In thin section we determined a compact shaly and sideritic siltstone with abundant thin shaly and coaly interbeds.

Sample 79-1, 79-80 cm (852.79-852.80 m sub-bottom). This pebble has a subangular shape with dimensions $2 \times 1.5 \times 1.5 \mathrm{~cm}$; it is medium greenish brown. In thin section we determined a compact shaly siltstone slightly micaceous and glauconitic, with abundant brown to black organic debris scattered between the silt grains.

\section{Site 550}

In Hole 550, one ice-rafted pebble was found within the single core taken at the seabed (Pleistocene):

Sample 1-4, $60 \mathrm{~cm}$ (5.10 m sub-bottom) (Plate 3). This pebble has a subangular shape with dimensions 2.5 $\times 2 \times 2 \mathrm{~cm}$; it is dark brown with light gray interbeds. In thin section we determined a very fresh and unaltered gneiss with biotite. It has a medium- to coarse-grained granoblastic and bedded structure, and is made up of quartz $(40 \%)$, microcline $(20 \%)$, oligoclase $(20 \%)$, and biotite $(20 \%)$; accessory minerals are muscovite, apatite, and sphene.

\section{Conclusions}

Pebbles found in the sediments of Holes 548, 549, and 550 have three origins: they are either ice-rafted or detrital, or have a diagenetic origin.

Ice-rafted pebbles (10) occur in upper Pliocene and Pleistocene sediments cored in Hole 548 and in Pleistocene seabed sediments cored in Hole 550. Most (6) are sedimentary rocks; the rest have a metamorphic (2) or igneous (2) origin.

In Hole 548, where ice-rafted pebbles are the most abundant, the lowermost one was recovered from upper 

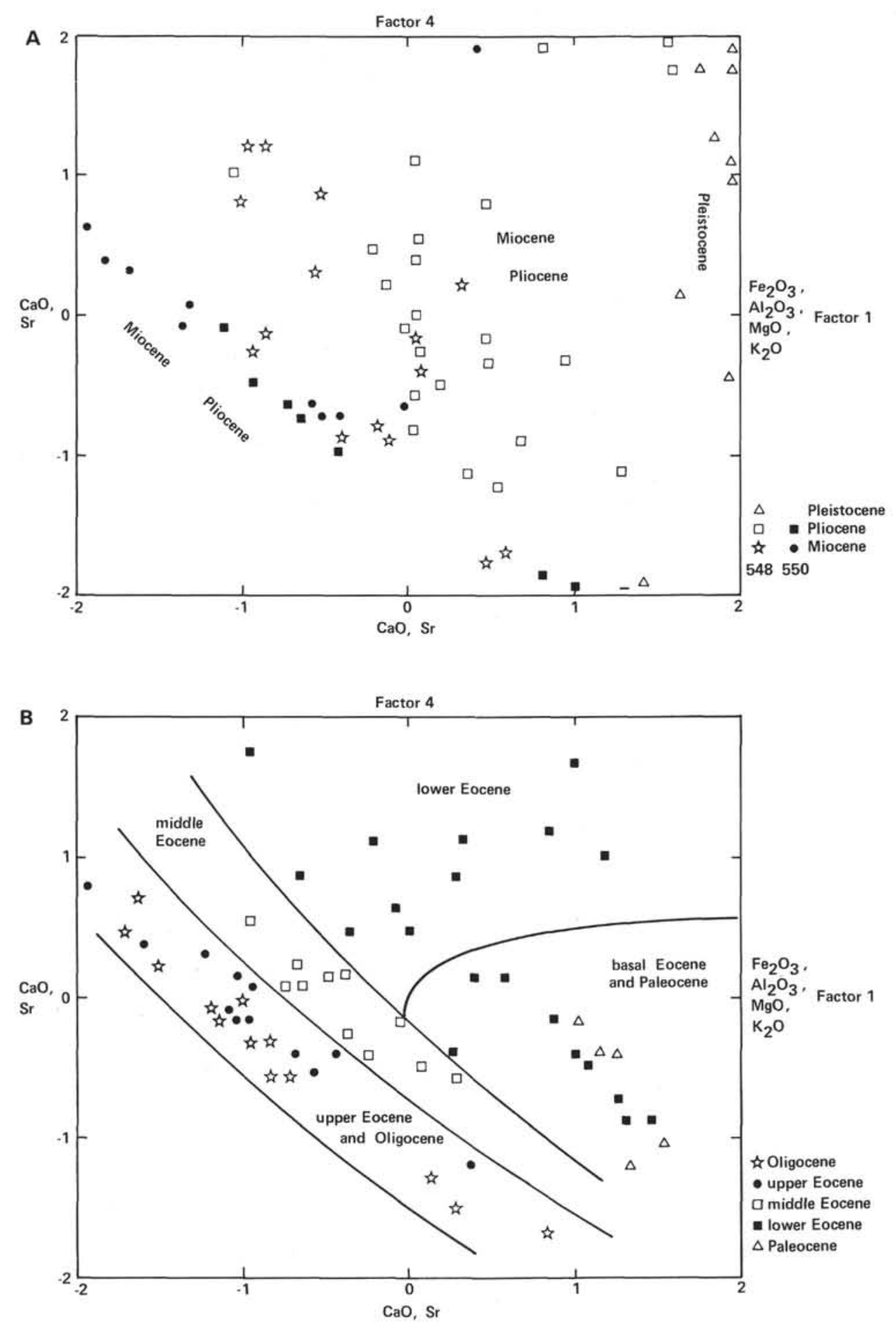

Figure 9. Factor-scores plotting of $1 \times 4$ lithological factors. A. Miocene to Pleistocene. B. Paleocene to Oligocene.

Pliocene sediments. This allows us to place the beginning of glaciation quite accurately in the stratigraphic column.

It must be also pointed out that these pebbles occur in a series where the mineralogical composition of deposits, and particularly of their clay fractions, under- goes numerous variations related to glaciations and climatic fluctuations.

Ice-rafted pebbles such as those described here were previously described by Auffret et al. (1979), but his samples were all from dredge samples collected around DSDP Hole 440. 

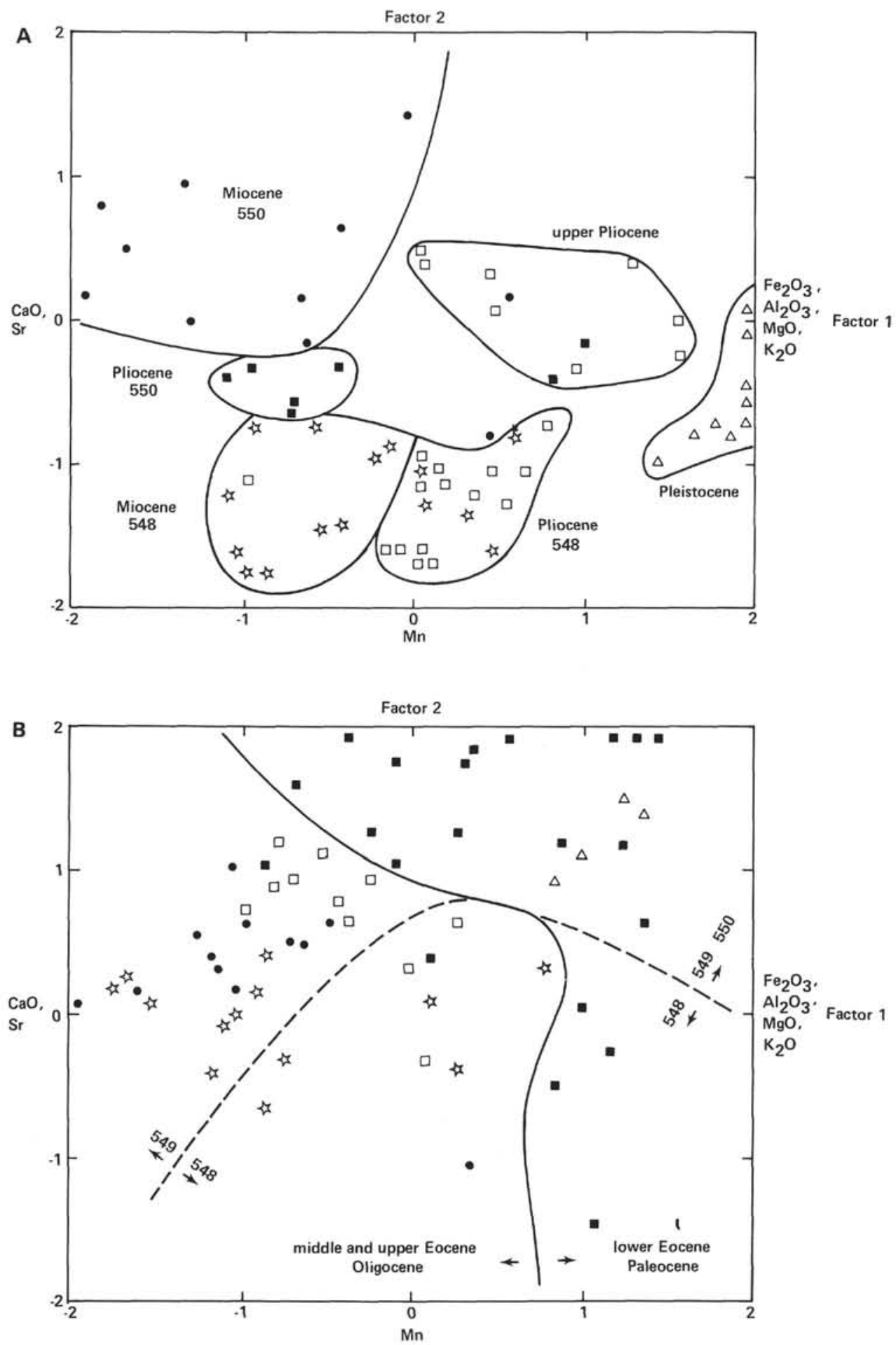

Figure 10. Factor-scores plotting of 1 lithological $\times 2$ Mn factors. A. Miocene to Pleistocene. B. Paleocene to Oligocene.

Two detrital pebbles occur in Lower Cretaceous sediments cored in Hole 549, associated with terrigenous sediments; both have a sedimentary origin.

Finally, the pebble found in Hole 548, Core 20, within upper Pliocene to upper Miocene sediments, is interpreted as an in situ diagenetic nodule related to local organic activity and reducing conditions.

\section{GENERAL CONCLUSIONS}

The mineralogical, geochemical, and petrographic studies of Leg 80 samples contribute, we believe, to a better understanding of the evolution of the northeastern Atlantic province during the Cenozoic. Mutually complementary data provided by the different techniques used 
in this study show that this evolution-although on the whole progressive from the Paleocene to the Pleistocene (e.g., the general decrease of the smectite/[illite + chlorite] ratio)-was nevertheless marked by "crises," recorded particularly at the middle/upper Miocene boundary and toward the top of the Pliocene. These crises, occurring at the same time as the previously described worldwide events, may indicate that their causes were not local but climatic and/or hydrological.

\section{ACKNOWLEDGMENTS}

We are very grateful to P. de Graciansky, Co-Chief Scientist of Leg 80 , for choosing the samples we have examined, for his scientific advice, and for enabling us to participate in this study through the support of the A.T.P. number A 15066 "Géologie et Géophysique des Océans." We are also very grateful to M. Hoffert and B. Biju-Duval for reviewing the manuscript. The mineralogical study carried out in Talence by the I.G.B.A. has also been financed by the A.T.P. "Géologie et Géophysique des Océans" (IPOD 82-84-Contribution de la connaissance des paléoenvironnements à l'établissement d'une biostratigraphie du Quaternaire marin de l'Atlantique nord-oriental).

\section{REFERENCES}

Alvinerie, J., Caralp, M., Latouche, C., Moyes, J., and Vigneaux, M., 1978. Apport à la connaissance de la paléohydrologie de l'Atlantique nord-oriental pendant le Quaternaire terminal. Oceanol. Acta, 1(1):87-98.

Auffret, G. A., Pastouret, L., Cassat, G., de Charpal, O., Cravatte, J., and Guennoc, P., 1979. Dredged rocks from the Armorican and Celtic margins. In Montadert, L., Roberts, D. G., et al., Init. Repts. DSDP, 48: Washington (U.S. Govt. Printing Office), 995-1013.

Berggren, W., and Holister, C. D., 1974. Paleogeography, paleobiogeography and the history of circulation in the Atlantic Ocean. Studies in Paleoceanography. Soc. Econ. Paleontol. Mineral. Publ., 20:126-186.

Biscaye, P. E., 1965. Mineralogy and sedimentation of Recent deep sea clay in the Atlantic Ocean and adjacent seas and oceans. Geol. Soc. Am. Bull., 76:803-832.

Blank, R. G., and Margolis, S. V., 1975. Pliocene climatic and glacial history of Antarctica, as revealed by South-East Indian Ocean deep seas cores. Geol. Soc. Am. Bull., 86:1058-1066.

Brown, G., 1961. The X-Ray Identification and Crystal Structures of Clay Minerals: London (Mineralogical Society).

Cassat, G., 1979. X-ray mineralogy from Holes 399, 400, 400A, 401, 402 and 402 A of Bay of Biscay. In Montadert, L., Roberts, D. G., et al., Init. Repts. DSDP, 48: Washington (U.S. Govt. Printing Office), 649-663.

Cavelier, C., Chateauneuf, J. J., Pomerol, C., Rabussier, D., Renard, M., and Vergnaud Grazzini, C., 1981. The geological events at the Eocene/Oligocene boundary. Palaeogeogr., Palaeoclimatol., Palaeoecol., 36:223-248.

Chamley, H., 1979. North Atlantic clay sedimentation and paleoenvironment since the Late Jurassic. In Talwani, M., Hay, W., and Ryan, W. B. F. (Eds.), Deep Drilling Results in the Atlantic Ocean: Continental Margins and Paleoenvironment, pp. 342-361.

Chamley, H., Debrabant, P., Foulon, J., Giroud d'Argoud, G., Latouche, C., Maillet, N., Maillot, H., and Sommer, F., 1979. Mineralogy and geochemistry of Cretaceous and Cenozoic Atlantic sediments off the Iberian Peninsula (Site 398, DSDP Leg 47B). In Sibuet, J.-C., Ryan, W. B. F., et al., Init. Repts. DSDP, 47, Pt. 2: Washington (U.S. Govt. Printing Office), 429-449.
Courtois, C., Chamley, H., 1978. Terres rares et minéraux argileux dans le Crétacé et le Cénozoïque de la marge Atlantique orientale. C. R. Acad. Sci. Paris, 286:1215-1218.

Fan, P., and Zemmels, I., 1972. X-ray mineralogy studies. In Berggren, W. A., Laughton, A. S., et al., Init. Repts. DSDP, 12: Washington (U.S. Govt. Printing Office), 1127-1154.

Griffin, J. J., Windom, H., and Goldberg, E. D., 1968. The distribution of clay minerals in the world Ocean. Deep Sea Res., 15: 433-459.

Grousset, F., Latouche, C., and Maillet, N., in press. Clay minerals as indicators of wind and current contribution to post-glacial sedimentation on the Azores/Iceland Ridge. Clay Min., 18.

Harman, H. H., 1960. Modern Factor Analysis: Chicago (University of Chicago Press).

Kaiser, H. F., 1958. The varimax criterion for analytical rotation in factor analysis. Psychometrika, 23:187-200.

Latouche, C., and Maillet, N., 1978. Minéralogie des dépôts des sites DSDP, IPOD, 398 (Leg 47B) et 403, 404, 405, 406 (Leg 48). Implications paléoclimatiques et paléohydrologiques. Bull. Soc. Geol. France, 7(4):411-427.

1979. X-ray mineralogy studies, Leg 48-Rockall region (Sites 403, 404, 405, and 406). In Montadert, L., Roberts, D. G., et al., Init. Repts. DSDP, 48: Washington (U.S. Govt. Printing Office), 665-676.

1980. Minéraux argileux et évolution des environnements sédimentaires du domaine Atlantique nord-oriental durant le Paléogène. Bull. Soc. Geol. France, 7(4):757-761.

1982. Essai sur l'utilisation des argiles comme témoins des climats néogènes dans la province Atlantique nord-orientale. Bull. Soc. Geol. France, 7(3):487-496.

Millot, G., 1964. Géologie des Argiles: Paris (Masson).

Paquet, H., 1969. Evolution géochimique des minéraux argileux dans les altérations et les sols des climats méditerranéens et tropicaux à saisons contrastées. Mem. Serv. Carte Geol. Alsace-Lorraine, Strasbourg, 30:1-212.

Rateev, M. A., Gorbunova, Z. N., Lisitzin, A. P., and Noson, G. I., 1969. Climatic zonality of the argillaceous minerals in world ocean sediments. Okeanology, 18:283-311.

Roberts, D. G., and Montadert, L., 1979a. Evolution of passive rifted margins. Perspective and retrospective of DSDP Leg 48. In Montadert, L., Roberts, D. G., et al., Init. Repts. DSDP, 48: Washington (U.S. Govt. Printing Office), 1143-1153.

1979b. Margin paleoenvironments of the northeast Atlantic. In Montadert, L., Roberts, D. G., et al., Init. Repts. DSDP, 48: Washington (U.S. Govt. Printing Office), 1099-1118.

Shackleton, N. J., and Kennett, J. P., 1975. Oxygen and carbon isotopic changes at late Cenozoic DSDP Site 284: implications for the glacial history of the northern hemisphere and Antarctica. In Kennett, J. P., Houtz, R. E., et al., Init. Repts. DSDP, 29: Washington (U.S. Govt. Printing Office), 801-807.

Talwani, M., and Eldholm, O., 1977. Evolution of the NorwegianGreenland Sea. Geol. Soc. Am. Bull., 88:969-999.

Thorez, J., 1975. Phyllosilicates and Clay Minerals. A Laboratory Handbook for Their X-Ray Analysis: Dison, Belgique (Lelotte).

van Andel, T. H., 1975. Mesozoic/Cenozoic calcite compensation depth and the global distribution of calcareous sediments. Earth Planet. Sci. Lett., 26:187-195.

Vergnaud Grazzini, C., Müller, C., Pierre, C., Létolle, R., and Peypouquet, J. P., 1979. Stable isotopes and Tertiary paleontological paleooceanography in the northeast Atlantic. In Montadert, L., Roberts, D. G., et al., Init. Repts. DSDP, 48: Washington (U.S. Govt. Printing Office), 475-491.

Date of Initial Receipt: November 22, 1982

Date of Acceptance: May 3, 1983 




Plate 1. Sample 548-2-5, $71-72 \mathrm{~cm}$, calk-alkaline syenite. Polarized light $(\times 40)$. 




Plate 2. Sample 548-11-3, 120-121 cm, calcareous biomicite. Natural light $(\times 40)$. 


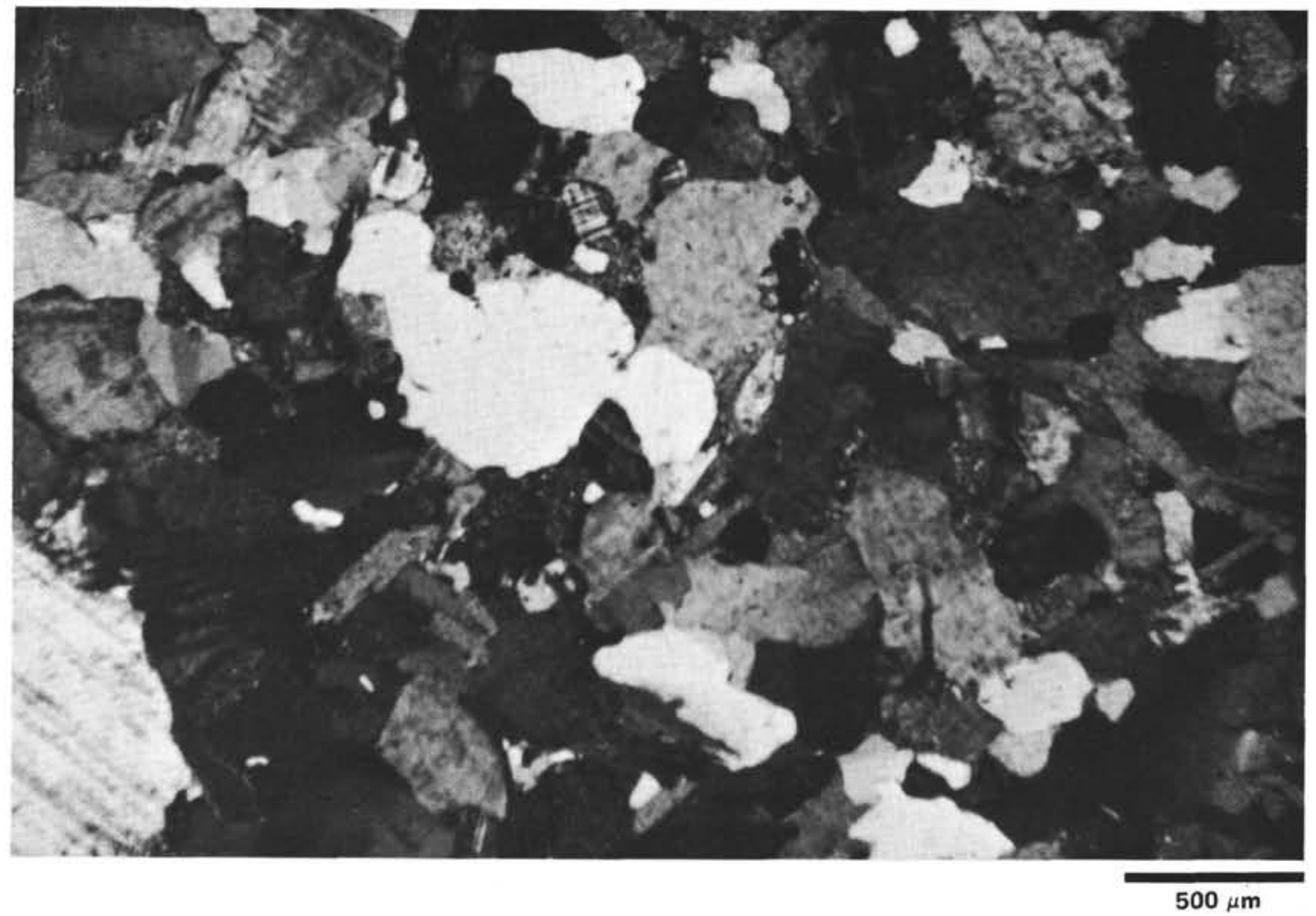

Plate 3. Sample 550-1-4, $60 \mathrm{~cm}$, gneiss with biotite. Polarized light $(\times 40)$. Gneiss with biotite. 